\title{
Duality for Vanishing Cycle Functors
}

\author{
By \\ Morihiko SAITo*
}

\section{Introduction}

Let $Y$ be a complex manifold, and $S$ an open disc. Put $X=Y \times S$, and identify $Y$ with $Y \times\{0\}$. Let $(M, F)$ be a holonomic filtered $\mathscr{D}_{X}$-Module, i.e. $M$ is holonomic and $G r^{F} M$ is coherent over $G r^{F} \mathscr{D}_{X}$, where $\mathscr{D}_{X}$ is the sheaf of holomorphic differential operators with $F$ the filtration by the degree of operator. We say that $(M, F)$ is Cohen-Macaulay, if $\mathrm{Gr}^{F} M$ is Cohen-Macaulay over $\operatorname{Gr}^{F} \mathscr{D}_{X}$, or equivalently

$$
\mathcal{E x t}_{G r^{F} \mathscr{D}_{X}}^{i}\left(G r^{F} M, G r^{F} \mathscr{D}_{X}\right)=0 \text { for } i \neq \operatorname{dim} X
$$

assuming $M$ holonomic. For $(M, F)$ holonomic Cohen-Macaulay, we define the dual $\boldsymbol{D}(M, F)$ by

$$
D(M, F)=\operatorname{Ext}_{\mathscr{D}_{X}}^{\operatorname{dim} X}\left((M, F),\left(\omega_{X}, F\right) \otimes \mathcal{O}_{X}\left(\mathscr{D}_{X}, F\right)\right)
$$

using a resolution of $(M, F)$ (and $\left(\omega_{X}, F\right)$ ), cf. 1.1, where $\omega_{X}$ is the sheaf of holomorphic differential forms of highest degree and $G r_{p}^{F} \omega_{X}=0$ for $p \neq 0$. Assume that $(M, F)$ has the filtration $V$ satisfying the conditions (1.2.1-7) in 1.2. Then we can define the vanishing cycle functors by

$$
\begin{aligned}
& \psi(M, F)=\bigoplus_{-1 \leq \alpha<0} G r_{a}^{V}(M, F[1]) \\
& \phi_{1}(M, F)=G r_{0}^{V}(M, F) .
\end{aligned}
$$

We assume that $(M, F)$ is holonomic Cohen-Macaulay, and $\psi(M, F), \phi_{1}(M, F)$ are holonomic Cohen-Macaulay filtered $\mathscr{D}_{Y}$-Modules. Then

Theorem (cf. 1.6). We have the functorial isomorphisms:

$$
\begin{aligned}
& \psi \mathbb{D}(M, F)=\mathbb{D} \psi(M, F)(1) \\
& \phi_{1} D(M, F)=\mathbb{D} \phi_{1}(M, F) .
\end{aligned}
$$

Received June 2, 1988.

* Research Institute for Mathematical Sciences, Kyoto University, Kyoto 606, Japan, and Institut des Hautes Etudes Scientifiques, Bures-sur-Yvette, 91440 France 
We also show that these duality isomorphisms are compatible with topological ones, i.e. by the natural isomorphisms $D R(\psi M)=\psi D R(M)[-1], D R\left(\phi_{1} M\right)=$ $\phi_{1} D R(M)[-1]$ in [S1, 3.4.12] (cf. also [M] [K2]) and $D R \circ \mathbb{D}=\mathbb{D} \circ D R$ in [S1, $2.4 .12]$ (cf. also [K4]), they correspond to the induced pairings $-{ }^{p} \psi \mathbb{S},{ }^{p} \phi_{1} \mathbb{S}$ in $[S 1,5.2 .3-4]$ on the vanishing cycles of $D R(M)$ and $D R(D M)$, where $S$ is the natural pairing of $D R(M)$ and $D R(\mathbb{D} M)$, cf. 2.2. Note that the arguments used in the proof of these results can be easily applied to the non filtered case, cf. 1.7, 2.4, and in the one dimensional case they were obtained in the Appendix of [S3]. In the normal crossing case, we calculate the vanishing cycle functors and the induced dualities on them explicitly, cf. $\S 3$. These results are used in the proof of [S2].

I would like to thank Prof. Kashiwara for useful discussions. Some part of this work was done during my stay at IHES. I would like to thank the staff of the institute for the hospitality.

\section{§1. Analytic Duality for Vamishing Cycle Functors}

1.1. Dual functor (cf. [S1]). Let $X$ be a complex manifold of dimension $n$, and $\mathscr{D}_{X}$ the sheaf of holomorphic differential operators with the filtration $F$ by the degree of operators. In this note we use the right $\mathscr{D}$-Modules, because they are more convenient to the definition of the dual functor.

Let $\operatorname{MF}\left(\mathscr{D}_{X}\right)$ be the category of filtered $\mathscr{D}_{X}$-Modules $(M, F)$ such that $F$ is exhaustive and $F_{p}=0$ for $p \ll 0$ locally on $X$, and $M G\left(\oplus_{p} F_{p} \mathscr{D}_{X}\right)$ the category of graded Modules $M$. over the graded algebra $\oplus_{p} F_{p} \mathscr{D}_{X}$ such that $M_{p}=0$ for $p \ll 0$ locally on $X$. By the natural functor $(M, F) \mapsto \bigoplus_{p} F_{p} M, M F\left(\mathscr{D}_{X}\right)$ is a full subcategory of $M G\left(\oplus_{p} F_{p} \mathscr{D}_{X}\right)$. We denote by $D_{\text {coh }}^{b} F\left(\mathscr{D}_{X}\right)$ the derived category of bounded complexes of filtered $\mathscr{D}_{X}$-Modules whose cohomologies in $M G\left(\oplus_{p} F_{p} M\right)$ are coherent over $\oplus_{p} F_{p} \mathscr{D}_{X}$.

We say that a filtered $\mathscr{D}_{X}$-Module $(M, F)$ is induced, if it is isomorphic to $(L, F) \otimes_{\mathcal{O}}\left(\mathscr{D}_{X}, F\right)$ for a filtered $\mathcal{O}_{X}$-Module $(L, F)$. For an induced filtered $\mathscr{D}_{X}$-Module $(M, F)$ we define a filtration $G$ by $G_{p} M=\left(F_{p} M\right) \mathscr{D}_{X}$ so that $G r_{p}^{G}$ $(M, F)$ is isomorphic to $G r_{p}^{F} L \otimes_{\mathcal{O}}\left(\mathscr{D}_{X}, F([p])\right.$, where $(F[p])_{i}=F_{i-p}$. We denote by $D_{\text {coh }}^{b} F_{i}^{f}\left(\mathscr{D}_{X}\right)$ the derived category of bounded complexes of induced filtered $\mathscr{D}_{X}$-Modules $\left(M^{\circ}, F\right)$ such that the filtration $G$ is finite locally on $X$ and $\mathcal{H}^{i} G r_{p}^{F} G r_{p}^{G} M^{\bullet}$ are coherent $\mathcal{O}_{X}$-Modules. Then we can show the equivalence of categories (cf. [S1, 2.1.16]):

$$
D_{\mathrm{coh}}^{b} F_{i}^{f}\left(\mathscr{D}_{X}\right) \underset{\mathrm{coh}}{\sim} F\left(\mathscr{D}_{X}\right) .
$$


Let $\omega_{X}$ be the dualizing sheaf of $X$, i.e. it is canonically isomorphic to $\Omega_{X}^{n}$ the sheaf of holomorphic $n$-forms and has the structure of right $\mathscr{D}_{X}$-Module. We take $\omega_{X}[n] \rightarrow K_{X}$ a resolution by a complex of right $\mathscr{D}_{X}$-Modules whose components are injective $\mathcal{O}_{X}$-Modules. (For $K_{X}$ we can take a bounded complex by a result of V.D. Golvin, Soviet Math. Dokl. 16 (1975)p. 854.) For $(M, F) \in$ $D_{\text {coh }}^{b} F_{i}^{f}\left(\mathscr{D}_{X}\right)$, put

$$
\boldsymbol{D}(M, F)=\operatorname{Ham}_{\mathscr{D}}^{F}\left((M, F),\left(K_{X}, F\right) \otimes_{\mathcal{O}}\left(\mathscr{D}_{X}, F\right)\right),
$$

where the filtration $F$ on $K_{X}$ is trivial, i.e. $G r_{p}^{F} K_{X}=0$ for $p \neq 0$, so that $F_{p}\left(K_{X} \otimes\right.$ $\left.\mathscr{D}_{X}\right)=K_{X} \otimes F_{p} \mathscr{D}_{X}$, and $K_{X} \otimes \mathcal{O}_{X}$ is a right $\mathscr{D}_{X}$-bi-Module having an involution which is the identity on $\mathcal{O}_{X}$ and exchanges the two structures of right $\mathscr{D}_{X^{-}}$ Module (cf. [S1, 2.4.2]). Here Ham $^{F}$ means the union (or inductive limit) of morphisms which preserve the filtration up to shifts so that $\boldsymbol{D}(M, F)$ becomes a complex of filtered $\mathscr{D}_{X}$-Modules whose components are induced, cf. [S1, 2.4.3]. Moreover $\mathbb{D}(M, F)$ belongs to $D_{\text {coh }}^{b} F_{i}^{f}\left(\mathscr{D}_{X}\right)$ essentially, i.e. $\mathcal{H}^{j} G r^{F}$ is zero for $j \gg 0$, and we get the dual functor:

$$
\boldsymbol{D}: D_{\text {coh }}^{b} F_{i}^{f}\left(\mathscr{D}_{X}\right) \rightarrow D_{\text {coh }}^{b} F_{i}^{f}\left(\mathscr{D}_{X}\right)^{\circ}, \quad \text { cf. [loc. cit] . }
$$

Note that $D$ is independent of the choice of $K_{X}$, because we can take for $K_{X}$ a resolution by injective $\mathscr{D}_{X}$-Modules (so that a morphism of complexes between two resolutions is constructed.) By (1.1.1) we get also

$$
D: D_{\text {coh }}^{b} F\left(\mathscr{D}_{X}\right) \rightarrow D_{\text {coh }}^{b} F\left(\mathscr{D}_{X}\right)^{\circ}
$$

compatible with (1.1.3) by (1.1.1). We denote by $M F_{\text {hol }}\left(\mathscr{D}_{X}\right)$ the full subcategory of $M F_{\text {coh }}\left(\mathscr{D}_{X}\right)$ consisting of holonomic filtered $\mathscr{D}_{X}$-Modules, where a filtered $\mathscr{D}_{X}$-Module $(M, F)$ is called holonomic, if $M$ is holonomic and $G r^{F} M$ is coherent over $G r^{F} \mathscr{D}_{X}$. Then $M F_{\text {hol }}\left(\mathscr{D}_{X}\right)$ is a full subcategory of $D_{\text {coh }}^{b} F\left(\mathscr{D}_{X}\right)$, and for $(M, F) \in M F_{\text {hol }}\left(\mathscr{D}_{X}\right), D(M, F)$ belongs to $M F_{\text {hol }}\left(\mathscr{D}_{X}\right)$ (i.e. the differential is strictly compatible with $F$ ) iff $G r^{F} M$ is a Cohen-Macaulay $G r^{F} \mathscr{D}_{X}$-Module, i.e.

$$
\mathcal{E}_{x t^{i}}^{i} r^{F} \mathscr{D}_{X}\left(G r^{F} M, G r^{F} \mathscr{D}_{X}\right)=0 \text { for } i \neq n .
$$

In fact for a local calculation of $\boldsymbol{D}(M, F)$ we can take a resolution of $(M, F)$ by finite free filtered $\mathscr{D}_{X}$-Modules locally on $X$ instead of taking the resolution $K_{X}$ of $\omega_{X}[n]$, so that $G r^{F}$ commute with the dual functor. Here the assumption $M$ holonomic implies $\mathbb{D} M \in M_{\text {hol }}\left(\mathscr{D}_{X}\right)$ and

$$
\mathcal{E x t}^{i} \mathscr{D}_{X}\left(M, \mathscr{D}_{X}\right)=0 \text { for } i \neq n, \mathrm{cf} .[\mathrm{K} 1] \text {. }
$$

We denote by $M F_{l l C M}\left(\mathscr{D}_{X}\right)$ the full subcategory of $M F_{\text {hol }}\left(\mathscr{D}_{X}\right)$ consisting of 
$(M, F)$ such that $G r^{F} M$ are Cohen-Macaulay, so that we have the dual functor

$$
\mathbb{D}: M F_{h C M}\left(\mathscr{D}_{X}\right) \rightarrow M F_{h C M}\left(\mathscr{D}_{X}\right)^{\circ} \text {. }
$$

1.2. Vanishing cycle functors. Let $Y$ be a complex manifold of dimension $n-1$, and put $X=Y \times S$, where $S$ is an open disc with the coordinate $t$, and $Y$ will be identified with $Y \times\{0\}$ in $X$. Let $V_{0} \mathscr{D}_{X}$ be the subalgebra of $\mathscr{D}_{X}$ generated by $\mathcal{O}_{X}, \mathscr{D}_{Y}$ and $s:=t \partial_{t}$. We define the increasing filtration $V$ of $\mathscr{D}_{X}$ by

$$
V_{i} \mathscr{D}_{X}=\left(V_{0} \mathscr{D}_{X}\right) t^{-i}(i \leqq 0), \sum_{0 \leqq j \leqq i}\left(V_{0} \mathscr{D}_{X}\right) \partial_{t}^{j}(i \geqq 0)
$$

Sometimes $V$ will be indexed by $\mathbb{Q}$ so that $V_{\alpha}=V_{i}$ for $i \leqq \alpha<i+1$ and $i \in \mathbb{Z}$. Let $M$ be a $\mathscr{D}_{X}$-Module. By Kashiwara [K2], there exists at most one increasing exhaustive filtration $V$ of $M$, indexed by $Q$ and satisfying:

$$
\begin{aligned}
& V_{\alpha} M \text { are coherent } V_{0} \mathscr{D}_{X} \text {-sub-Modules of } M, \\
& \left(V_{\alpha} M\right) V_{i} \mathscr{D}_{X} \subset V_{\alpha+i} M \text { for } \alpha \in \mathbb{Q}, i \in \mathbb{Z}, \\
& \left(V_{\alpha} M\right) t=V_{\alpha-1} M \text { for } \alpha<0, \\
& t \partial_{t}-\alpha \text { is nilpotent on } G r_{\alpha}^{V} M \text { for } \alpha \in \mathbb{Q} \text { locally on } Y,
\end{aligned}
$$

where we assume that $V$ is discretely indexed locally on $X$, i.e. there is locally a positive integer $m$ such that $V_{\alpha}=V_{i / m}$ for $i / m \leqq \alpha<(i+1) / m$, and put $V_{<\beta} M=$ $\cup_{\omega<\beta} V_{\alpha} M$ for $\beta \in \mathbb{R}$. Let $(M, F)$ be a holonomic filtered $\mathscr{D}_{X}$-Module. We assume that $M$ has the filtration $V$ satisfying (1.2.1-4) and

$$
\begin{aligned}
& t: F_{p} V_{\alpha} M \stackrel{\mathbb{F}_{p}}{\sim} V_{\alpha-1} M(\alpha<0), \\
& \partial_{t}: F_{p} G r_{\alpha}^{V} M \stackrel{\sim}{\rightarrow} F_{p+1} G r_{\alpha+1}^{V} M(\alpha>-1), \\
& G r_{\alpha}^{V}(M, F) \text { are holonomic filtered } \mathscr{D}_{Y^{-} \text {-Modules. }}
\end{aligned}
$$

Then we define the vanishing cycle functors of $(M, F)$ by:

$$
\begin{aligned}
& \psi(M, F)=\bigoplus_{-1 \leqq \alpha<0} G r_{\alpha}^{V}(M, F[1]), \psi_{1}(M, F)=G r_{-1}^{V}(M, F[1]), \\
& \psi_{\neq 1}(M, F)=\phi_{\neq 1}(M, F)=\bigoplus_{-1<\alpha<0} G r_{\alpha}^{V}(M, F[1]), \\
& \phi_{1}(M, F)=G r_{0}^{V}(M, F),
\end{aligned}
$$

and define can: $\psi_{1}(M, F) \rightarrow \phi_{1}(M, F)$ and $\operatorname{Var}: \phi_{1}(M, F) \rightarrow \psi_{1}(M, F[-1])$ by $\partial_{t}$ and $t$ so that Varocan $=N$ and can०Var $=N$, where $N:=t \partial_{t}-\alpha$ on $G r_{a}^{V} M$.

1.3. Filtered graded Modules. Let $M G F\left(G r^{V} \mathscr{D}_{X}\right)$ be the category of filtered Q-graded $G r^{V} \mathscr{D}_{X}$-Modules $\left(M_{\circ}, F\right)=\bigoplus_{\alpha \in Q}\left(M_{\alpha}, F\right)$ such that for some $m \in \mathbb{Z}$, $M_{\alpha}=0$ if $\alpha m \notin \mathbb{Z}, F_{p} M_{\alpha}=0$ for $p \ll 0$, locally on $Y$, and 


$$
\begin{aligned}
& G r_{-1}^{V} t:\left(M_{\alpha}, F\right) \stackrel{\sim}{\rightarrow}\left(M_{\alpha-1}, F\right) \text { for } \alpha<0, \\
& G r_{1}^{V} \partial_{t}:\left(M_{\alpha}, F\right) \stackrel{\sim}{\rightarrow}\left(M_{\alpha+1}, F[-1]\right) \text { for } \alpha>-1,
\end{aligned}
$$

where we assume that the filtration $F$ on each $M_{\alpha}$ is exhaustive and $\left(F_{p} M_{\alpha}\right)$ $F_{i} G r_{j}^{V} \mathscr{D}_{X} \subset F_{p+i} M_{\alpha+j}$. We denote by $M G F_{m c}\left(G r^{V} \mathscr{D}_{X}\right)$ (resp. $M G F_{m h}\left(G r^{V} \mathscr{D}_{X}\right)$ ) the full subcategory of $M G F\left(G r^{V} \mathscr{D}_{X}\right)$ defined by the conditions:

$$
G r_{0}^{V} t \partial_{t}-\alpha \text { is nilpotent on } M_{\alpha} \text { locally on } Y \text {, }
$$

$$
\left(M_{\alpha}, F\right) \text { are coherent (resp. holonimic) filtered } \mathscr{D}_{Y} \text {-Modules. }
$$

Here $\mathscr{D}_{Y}$ is naturally identified with a subalgebra of $G r_{0}^{V} \mathscr{D}_{X}=\mathscr{D}_{Y}[s]$. The full subcategory of $M G F_{m h}\left(G r^{V} \mathscr{D}_{X}\right)$ defined by the condition:

$$
G r^{F} M_{\alpha} \text { are Cohen-Macaulay over } G r^{F} \mathscr{D}_{Y},
$$

will be denoted by $M G F_{m h C M}\left(G r^{V} \mathscr{D}_{X}\right)$.

By definition $(M, F) \in M G F\left(G r^{V} \mathscr{D}_{X}\right)$ has the natural decomposition $(M, F)$ $=\bigoplus_{0 \geqq \alpha>-1}\left(M_{\cdot}^{(\alpha)}, F\right)$ such that $M_{\beta}^{(\alpha)}=0$ for $\alpha-\beta \notin Z$. We denote by $M G F$ $\left(G r^{V} \mathscr{D}_{X}\right)^{(\alpha)}$ the full subcategory of $M G F\left(G r^{V} \mathscr{D}_{X}\right)$ defined by the condition: $M_{\beta}$ $=0$ for $\alpha-\beta \notin Z$. Put $M G F_{m c}\left(G r^{V} \mathscr{D}_{X}\right)^{(\alpha)}=M G F_{m c}\left(G r^{V} \mathscr{D}_{X}\right) \cap M G F\left(G r^{V} \mathscr{D}_{X}\right)^{(\alpha)}$ (same for $m h$ and $m h C M$ ).

Let $\operatorname{MF}\left(\mathscr{D}_{Y} ; N\right)$ be the category of filtered $\mathscr{D}_{Y}$-Modules $(M, F)$ with an endomorphism $N:(M, F) \rightarrow(M, F[-1])$ in $M F\left(\mathscr{D}_{Y}\right)$, and $M F\left(\mathscr{D}_{Y}\right.$; can, Var) the category of pairs of filtered $\mathscr{D}_{Y^{-}}$-Modules $\left(M^{\prime}, F\right),\left(M^{\prime \prime}, F\right)$ with morphisms

$$
\text { can }\left(M^{\prime}, F\right) \rightarrow\left(M^{\prime \prime}, F[-1]\right) \text {, Var: }\left(M^{\prime \prime}, F\right) \rightarrow\left(M^{\prime}, F\right) \text { in } M F\left(\mathscr{D}_{Y}\right) \text {. }
$$

Then we have equivalences of categories:

$$
\begin{aligned}
& \Psi^{\alpha}: M G F\left(G r^{V} \mathscr{D}_{X}\right)^{(\alpha)} \underset{\longrightarrow}{\longrightarrow} M F\left(\mathscr{D}_{Y} ; N\right) \text { for }-1<\alpha<0, \\
& \Psi^{0}: M G F\left(G r^{V} \mathscr{D}_{X}\right)^{(0)} \stackrel{M}{\longrightarrow} M F\left(\mathscr{D}_{Y} ; \text { can, Var }\right)
\end{aligned}
$$

by assigning $\left(M_{\alpha} ; G r_{0}^{V} t \partial_{t}-\alpha\right)$ or $\left(M_{-1}, M_{0} ; G r_{1}^{V} \partial_{t}, G r_{-1}^{V} t\right)$ to $\left(M_{.}, F\right)$. We have also

$$
\begin{aligned}
& \Psi^{\alpha}: M G F_{m c}\left(G r^{V} \mathscr{D}_{X}\right)^{(\alpha)} \underset{\sim}{\longrightarrow} M F_{m c}\left(\mathscr{D}_{Y} ; N\right) \text { for }-1<\alpha<0, \\
& \Psi^{0}: M G F_{m c}\left(G r^{V} \mathscr{D}_{X}\right)^{(0)} \stackrel{\sim}{\rightarrow} M F_{m c}\left(\mathscr{D}_{Y} ; \text { can, Var }\right)
\end{aligned}
$$

(same for $m h$ and $m h C M$ ), where the right hand sides are the full subcategories defined by the conditions:

$N$ is nilpotent locally on $Y$,

(1.3.8) $(M, F)$ (or $\left.\left(M^{\prime}, F\right),\left(M^{\prime \prime}, F\right)\right)$ is a coherent (resp. holonomic, resp. holonomic and Cohen-Macaulay) filtered $\mathscr{D}_{Y}$-Module. 
Here $N=\operatorname{Var} \circ$ can on $M^{\prime}, N=$ canoVar on $M^{\prime \prime}$, and $(M, F)$ is called CohenMacaulay if $G r^{F} M$ is Cohen-Macaulay over $G r^{F} \mathscr{D}_{Y}$.

Let $D^{b} G F\left(G r^{V} \mathscr{D}_{X}\right)^{(\alpha)}$ be the derived category of bounded complexes of $M G F\left(G r^{V} \mathscr{D}_{X}\right)^{(\alpha)}$, and $D_{m c}^{b} G F\left(G r^{V} \mathscr{D}_{X}\right)^{(\alpha)}$ (resp. $D_{m h}^{b} G F\left(G r^{V} \mathscr{D}_{X}\right)^{(\alpha)}$ ) its full subcategory defined by the following conditions (compare to (1.3.3-4)):

$$
\begin{aligned}
& G r_{0}^{V} t \partial_{t}-\beta \in F_{1} G r_{0}^{V} \mathscr{D}_{X} \text { is nilpotent on } \mathcal{H}^{j}\left(\oplus_{p} F_{p} M_{\beta}\right) \\
& \text { locally on } Y \text {, } \\
& \mathcal{H}^{j}\left(\oplus_{p} F_{p} M_{\beta}\right) \text { are coherent over } \bigoplus_{p} F_{p} \mathscr{D}_{Y} \text { (and } \mathcal{H}^{j} M_{\beta} \\
& \text { are holonomic } \mathscr{D}_{Y} \text {-Modules). }
\end{aligned}
$$

Similarly we define $D^{b} F\left(\mathscr{D}_{Y} ; N\right), D^{b} F\left(\mathscr{D}_{Y} ;\right.$ can, Var $), D_{m c}^{b} F\left(\mathscr{D}_{Y} ; N\right)$, etc., so that the equivalences of categories (1.3.5-6) are extended to the equivalences between the derived categories.

We say that $\left(M_{0}, F\right) \in M G F\left(G r^{V} \mathscr{D}_{X}\right)$ is semi-free (or $S$-free), if it is isomorphic to $\bigoplus_{-1 \leqq \alpha \leqq 0}\left(M_{\alpha}^{\prime}, F\right) \otimes_{C} G r^{V}\left(\mathscr{D}_{S}, F\right)$ for filtered $\mathscr{D}_{Y}$-Modules $\left(M_{\alpha}^{\prime}, F\right)$, where $\left(M_{\alpha}^{\prime}, F\right) \otimes 1$ is of degree $\alpha$ and $M_{\alpha}^{\prime}=0$ except for a finite number of $\alpha$ locally on $Y$ by hypothesis. Here we use the natural isomorphism

$$
\operatorname{Gr}^{V}\left(\mathscr{D}_{X}, F\right)=\left(\mathscr{D}_{Y}, F\right) \otimes_{C} G r^{V}\left(\mathscr{D}_{S}, F\right)
$$

as filtered graded algebras.

We say that $\left(M_{0}, F\right) \in M G F\left(G r^{V} \mathscr{D}_{X}\right)$ is induced (resp. Y-induced), if it is isomorphic to $\bigoplus_{-1 \leqq \alpha \leqq 0}\left(L_{\alpha}, F\right) \otimes \Theta_{Y} G r^{V}\left(\mathscr{D}_{X}, F\right)$ (resp. $\left(M_{\alpha}, F\right)$ is isomorphic to $\left(L_{\alpha}, F\right) \otimes \mathcal{O}_{Y}\left(\mathscr{D}_{Y}, F\right)$ as filtered $\mathcal{O}_{Y}$-Modules with action of $N$ for $\left.-1 \leqq \alpha \leqq 0\right)$, where $\left(L_{\alpha}, F\right)$ are filtered $\mathcal{O}_{Y}$-Modules (resp. filtered $\mathcal{O}_{Y}$-Modules with endomorphism $\left.N:\left(L_{\alpha}, F\right) \rightarrow\left(L_{\alpha}, F[-1]\right)\right)$ and they are zero except for a finite number of $\alpha$ locally on $Y$. Here $N$ on $M_{\alpha}$ is defined by $t \partial_{t}-\alpha$. For an induced (resp. a $Y$-induced) filtered graded Module $\left(M_{\bullet}, F\right)$, we define the filtration $G$ by

$$
G_{p} M_{\circ}=\left(F_{p} M_{0}\right) G r^{v} \mathscr{D}_{X} \quad\left(\text { resp. } G_{p} M_{\circ}=\left(F_{p} M_{\circ}\right) \mathscr{D}_{Y}\right)
$$

so that $G r_{p}^{G}\left(M_{0}, F\right)$ is isomorphic to $\bigoplus_{-1 \leqq \alpha \leqq 0} G r_{p}^{F} L_{\alpha} \otimes \mathcal{O}_{Y} G r^{V}\left(\mathscr{D}_{X}, F[p]\right)$ (resp. $\operatorname{Gr}_{p}^{G}\left(M_{\alpha}, F\right)$ is isomorphic to $G r_{p}^{F} L_{\alpha} \otimes \mathcal{O}_{Y}\left(\mathscr{D}_{Y}, F[p]\right)$ for $\left.-1 \leqq \alpha \leqq 0\right)$. We say that an induced (resp. $Y$-induced) filtered graded Module is quasi-finite, if $G$ is a finite filtration on $M_{\text {。 }}$ (resp. $M_{\alpha}$ for $-1 \leqq \alpha \leqq 0$ ) locally on $Y$. Note that an induced filtered graded Module is $Y$-induced, but it is not necessarily quasi-finite as a $Y$-induced Module, even if it is so as an induced Module, because the filtration $F$ on $L_{\alpha} \otimes_{C} G r_{0}^{V} \mathscr{D}_{S}$ is not finite, if $L_{\alpha} \neq 0$.

Let $D^{b} G F_{s f}\left(G r^{V} \mathscr{D}_{X}\right)$ (resp. $D^{b} G F_{i}\left(G r^{V} \mathscr{D}_{X}\right)$ or $D^{b} G F_{Y i}\left(G r^{V} \mathscr{D}_{X}\right)$ ) be the de- 
rived category of bounded complexes of semi-free (resp. induced or $Y$-induced) filtered graded Modules. Let $D^{b} G F_{i}^{f}\left(G r^{V} \mathscr{D}_{X}\right)$ (or $D^{b} G F_{Y}^{f}\left(G r^{V} \mathscr{D}_{X}\right)$ ) be that of quasi-finite induced (or $Y$-induced) filtered graded Modules. We define the full subcategory $D_{m c}^{b} G F_{s f}\left(G r^{V} \mathscr{D}_{X}\right)$ (resp. $D_{m c}^{b} G F_{i}^{f}\left(G r^{V} \mathscr{D}_{X}\right)$ or $D_{m c}^{b} G F_{Y i}^{f}\left(G r^{V} \mathscr{D}_{X}\right)$ ) by the cohomological conditions (1.3.9-10) (resp. (1.3.9) and (1.3.11) below):

$$
\mathscr{H}^{j} G r_{p}^{F} G r_{p}^{G} M_{\alpha} \text { are coherent } \mathcal{O}_{Y} \text {-Modules. }
$$

Let $D_{m k}^{b} G F_{s f}\left(G r^{V} \mathscr{D}_{X}\right)$, etc. be the full subcategory defined by adding the cohomologically holonomic condition (i.e. $\mathscr{H}^{j} M_{\alpha}$ are holonomic $\mathscr{D}_{Y}$-Modules). We also define the full subcategories $D_{m c}^{b} G F_{s f}\left(G r^{V} \mathscr{D}_{X}\right)^{(\alpha)}$, etc. by adding the condition: $M_{\boldsymbol{\beta}}=0$ for $\alpha-\beta \notin \boldsymbol{Z}$, so that

$$
D_{m c}^{b} G F_{s f}\left(G r^{V} \mathscr{D}_{X}\right)=\oplus_{-1<\alpha \leq 0} D_{m c}^{b} G F_{s f}\left(G r^{V} \mathscr{D}_{X}\right)^{(\alpha)}
$$

(same for $m h$ and $i, Y i$, etc.). For a complex of filtered graded Module $\left(M_{\bullet}, F\right)$, let $\left(M_{\bullet}, F\right)=\oplus_{-1<\alpha \leqq 0}\left(M_{\bullet}, F\right)^{(\alpha)}$ be the decomposition in (1.3.12). If $\left(M_{\bullet}, F\right)$ is induced, $G r_{p}^{G}\left(M_{.}, F\right)^{(0)}$ has the subcomplex generated by $F_{p} G r_{p}^{G} M_{0}$ over $G r^{V} \mathscr{D}_{X}$, and this subcomplex and its quotient complex will be denoted respectively by $G r_{p}^{G}\left(M_{.}, F\right)^{[0]}$ and $G r_{p}^{G}\left(M_{.}, F\right)^{[-1]}$, so that

$$
G r_{p}^{G}\left(M_{.}^{j}, F\right)^{[a]}=G r_{p}^{F} L_{a}^{j} \otimes \mathcal{O}_{Y} G r^{V}\left(\mathscr{D}_{X}, F[p]\right) \text { for } a=-1,0
$$

if $\left(M_{.}^{j}, F\right)=\oplus_{-1 \leqq \alpha \leqq 0}\left(L_{\alpha}^{j}, F\right) \otimes \mathcal{O}_{Y} G r^{V}\left(\mathscr{D}_{X}, F\right)$. Here note that $G r_{p}^{G}(M, F)^{(\alpha)}$ and $G r_{p}^{G}\left(M_{\bullet}, F\right)^{[a]}$ are filtered acyclic, if so is $\left(M_{.}, F\right)$, cf. [S1, 2.1.11]. We also check that $D^{b} G F_{i}^{f}\left(G r^{V} \mathscr{D}_{X}\right)$ is a full subcategory of $D^{b} G F_{i}\left(G r^{V} \mathscr{D}_{X}\right)$, i.e.

(1.3.14) $D^{b} G F_{i}^{f}\left(G r^{V} \mathscr{D}_{X}\right)$ is equivalent to the full subcategory defined by the cohomological condition: $\mathscr{H}^{j} G r_{p}^{G}\left(M_{\bullet}, F\right)$ are filtered acyclic for $p \gg 0$ locally on $Y$ (same for $Y$-induced Modules by replacing $\mathscr{H}^{j} G r_{p}^{G}\left(M_{\bullet}, F\right)$ with $\left.\mathscr{H}^{j} G r_{p}^{G}\left(M_{\alpha}, F\right)(-1 \leqq \alpha \leqq 0)\right)$,

cf. [S1, 2.1.13]. Then by the lemma 1.4 below, we get the equivalences of categories:

$$
\begin{array}{cc}
D_{m c}^{b} G F_{i}^{f}\left(G r^{V} \mathscr{D}_{X}\right) & \sim \\
\downarrow l & D_{m c}^{b} G F_{Y i}^{f}\left(G r^{V} \mathscr{D}_{X}\right) \\
D_{m c}^{b} G F_{s f}\left(G r^{V} \mathscr{D}_{X}\right) & \stackrel{\downarrow l}{\rightarrow} D_{m c}^{b} G F\left(G r^{V} \mathscr{D}_{X}\right)
\end{array}
$$

which remains valid if we add the upper suffix $(\alpha)$.

In fact, in the proof of (1.1.1), we used the canonical resolution by bounded complex of induced Modules (cf. [S1, 2.1.6]) which is also valid in the case where the filtered Modules have the endomorphism $N$ or the morphisms can, 
Var. Therefore we get the equivalences of categories forgetting the cohomological conditions for $m c$ and $f$. The coherence condition (1.3.11) for $\left(M_{\circ}, F\right) \in$ $D^{b} G F_{i}^{f}\left(G r^{V} \mathscr{D}_{X}\right)$ implies the coherence of $\mathcal{H}^{j}\left(\oplus_{p} F_{p} M_{\beta}\right)$ over $\oplus_{p} F_{p} G r_{0}^{V} \mathscr{D}_{X}$ (using the filtration $G$ and (1.3.13)), which implies (1.3.10), combined with (1.3.9). Thus we get (1.3.15) by the construction of 1.4 below and the proof of $[\$ 1,2.1 .16]$. Here note that (1.3.15) is valid by replacing the bounded condition $b$ by + , - or omitting it. Therefore we get

$$
\begin{aligned}
& D_{m c}^{b} G F_{i}^{f}\left(G r^{V} \mathscr{D}_{X}\right) \text { is equivalent to the full subcategory of } \\
& D_{m c}^{+} G F_{i}^{f}\left(G r^{V} \mathscr{D}_{X}\right) \text { defined by the cohomological condition: } \\
& \mathscr{H}^{j}\left(M_{\bullet}, F\right) \text { is filtered acyclic for } j \gg 0,
\end{aligned}
$$

which will be used in the definition of the dual functor.

1.4. Lemma. For a filtered graded Module $\left(M_{0}, F\right)$, let $\left(M_{0}, F\right)=\oplus\left(M_{0}, F\right)^{(\alpha)}$ be the decomposition as in (1.3.12), and put $\left(M_{\alpha}, F\right)_{\beta}^{S}:=\left(M_{\alpha}, F\right) \otimes_{C} G r^{V}\left(\mathscr{D}_{S}, F\right)$ $\in M G F_{s f}\left(G r^{V} \mathscr{D}_{X}\right)$, where the degree of $\left(M_{\alpha}, F\right) \otimes 1$ is $\beta$. Then we have the canonical semi-free resolution:

$$
\begin{aligned}
& 0 \rightarrow\left(M_{\alpha}, F[1]\right)_{\alpha}^{S} \stackrel{u}{\rightarrow}\left(M_{\alpha}, F\right)_{\alpha}^{S} \stackrel{v}{\rightarrow}\left(M_{\circ}, F\right)^{(\alpha)} \rightarrow 0 \quad(-1<\alpha<0), \\
& 0 \rightarrow\left(M_{0}, F\right)_{-1}^{S} \oplus\left(M_{-1}, F[1]\right)_{0}^{S} \stackrel{u^{\prime}}{\rightarrow}\left(M_{-1}, F\right)_{-1}^{S} \oplus\left(M_{0}, F\right)_{0}^{S} \stackrel{v^{\prime}}{\rightarrow}\left(M_{0}, F\right)^{(0)} \rightarrow 0,
\end{aligned}
$$

where the morphisms are defined by

$$
\begin{aligned}
& u(m \otimes P)=N m \otimes P-m \otimes(s-\alpha) P, \quad v(m \otimes P)=m P, \\
& u^{\prime}(m \otimes P, n \otimes Q)=\left(\operatorname{Var} m \otimes P-n \otimes \partial_{t} P, \operatorname{can} n \otimes Q-m \otimes t P\right), \\
& v^{\prime}(m \otimes P, n \otimes Q)=m P+n Q .
\end{aligned}
$$

Here can, Var and $N$ are defined by $\partial_{t}, t$ and $s-\alpha$ with $s=t \partial_{t}$.

Proof. We show the case $\alpha=0$. The remaining case is rather easy. By $(1.3 .1-2)$ it is enough to show the degree -1 and 0 part. We show the degree -1 part. The other case is similarly checked. The graduation of the degree -1 part of $u^{\prime}$ is

$$
\begin{aligned}
& G r_{p}^{F}\left(M_{0}\left[s^{\prime}\right]\right) \oplus G r_{p-1}^{F}\left(M_{-1}\left[s^{\prime}\right]\right) \rightarrow G r_{p}^{F}\left(M_{-1}\left[s^{\prime}\right]\right) \oplus G r_{p}^{F}\left(M_{0}\left[s^{\prime}\right]\right) \\
& U \\
& \left(m \otimes s^{\prime i}, n \otimes s^{\prime j}\right) \mapsto\left(\operatorname{Var} m \otimes s^{\prime i}-n \otimes s^{\prime j+1}, \operatorname{can} n \otimes s^{\prime j}-m \otimes s^{\prime i}\right)
\end{aligned}
$$

for $m \in G r_{p-i}^{F} M_{0}, n \in G r_{p-1-j}^{F} M_{-1}$, where $s^{\prime}:=s+1$ and $G r_{p}^{F}\left(M_{a}\left[s^{\prime}\right]\right)=\bigoplus_{i} G r_{p-i}^{F}$ $M_{a} \otimes s^{\prime i}$. We define the finite decreasing filtration $W$ on $G r_{p}^{F} M_{a}$ and $G r_{p}^{F}\left(M_{a}\left[s^{\prime}\right]\right)$ by 


$$
\begin{aligned}
& W^{2 i} G r_{p}^{F} M_{a}=N^{i} G r_{p-i}^{F} M_{a}, \quad W^{2 i+1} G r_{p}^{F} M_{-1}=\operatorname{Var} N^{i} G r_{p-i}^{F} M_{0}, \\
& W^{2 i+1} G r_{p}^{F} M_{0}=\operatorname{can} N^{i} G r_{p-i-1}^{F} M_{-1} \text { for } i \in \mathbb{N}, \\
& W^{i} G r_{p}^{F}\left(M_{a}\left[s^{\prime}\right]\right)=\bigoplus_{j}\left(W^{i} G r_{p-j}^{F} M_{a}\right) \otimes s^{\prime j} \text { for } i \in \mathbb{Z},
\end{aligned}
$$

where $a=-1,0$. Then $G r_{W} G r^{F}$ of $u^{\prime}, v^{\prime}$ become $s^{\prime} \oplus$ id and $\mathrm{pr}+0$, where pr: $G r_{p}^{F}\left(M_{a}\left[s^{\prime}\right]\right) \rightarrow G r_{p}^{F} M_{a}$ is the natural projection and 0 is the zero map. Therefore we get the assertion.

1.5. Dual functor on the filtered graded Modules. Let $\omega_{Y}\left[d_{Y}\right] \rightarrow K_{Y}$ be a resolution as right $\mathscr{D}_{Y}$-Modules such that $K_{Y}^{i}$ are injective $\mathscr{D}_{Y}$-Modules. Then $\omega_{Y}$ $\otimes \mathcal{O}_{Y} G r^{V} \mathscr{D}_{X}$ and $K_{Y}^{i} \otimes \mathcal{O}_{Y} G r^{V} \mathscr{D}_{X}$ have the structure of graded right $G r^{V} \mathscr{D}_{X}$-biModule so that we have the graded quasi-isomorphism:

$$
\omega_{Y}\left[d_{Y}\right] \otimes \mathcal{O}_{Y} G r^{V} \mathscr{D}_{X} \rightarrow K_{Y} \otimes \mathcal{O}_{Y} G r^{V} \mathscr{D}_{X}
$$

Here the twisted (i.e. not by multiplication from the right) action of $\operatorname{Gr}^{V} \mathscr{D}_{X}$ is defined by

$$
\begin{aligned}
& (m \otimes P) t(g)=m g \otimes P=m \otimes g P \text { for } \quad g \in \mathcal{O}_{Y} \\
& (m \otimes P) t(v)=m v \otimes P-m \otimes v P \text { for } \quad v \in \Theta_{Y} \\
& (m \otimes P) t\left(G r^{v} t\right)=m \otimes G r^{V} t P \\
& (m \otimes P) t\left(G r^{V} \partial_{t}\right)=-m \otimes G r^{v} \partial_{t} P
\end{aligned}
$$

where $m \in \omega_{Y}$ and $P \in G r^{V} \mathscr{D}_{X}$, and the degree of $\omega_{Y} \otimes G r_{i}^{V} \mathscr{D}_{X}$ is $i-1$ (same for $\left.K_{Y}^{i}\right)$. ( $t(*)$ means the twisted action.) Then for $\left(M_{\bullet}, F\right) \in D^{b} G F_{i}^{f}\left(G r^{V} \mathscr{D}_{X}\right)^{(\alpha)}$, we define the dual by:

$$
\mathbb{D}\left(M_{\bullet}, F\right)=\operatorname{Ham}_{G r^{v} \mathscr{D}_{X}}^{F}\left(\left(M_{\bullet}, F\right),\left(K_{Y}[1], F\right) \otimes \mathcal{O}_{Y} G r^{v}\left(\mathscr{D}_{X}, F\right)\right),
$$

where $\operatorname{Ham}^{F}$ is as in (1.1.2) and the filtration $F$ on $K_{Y}[1]$ is trivial. If $\left(M_{\bullet}, F\right)=$ $\left(L_{\alpha}, F\right) \otimes \mathcal{O}_{Y} G r^{V}\left(\mathscr{D}_{X}, F\right) \in M G F_{i}^{f}\left(G r^{V} \mathscr{D}_{X}\right)$ for $-1 \leqq \alpha \leqq 0$, we have

$$
\begin{aligned}
& \mathbb{D}\left(M_{\bullet}, F\right)=\operatorname{Ham}_{\Theta_{Y}}^{F}\left(\left(L_{\alpha}, F\right),\left(K_{Y}[1], F\right)\right) \otimes \mathcal{O}_{Y} G r^{V}\left(\mathscr{D}_{X}, F\right) \\
& G r_{p}^{F} \operatorname{Ham}_{\Theta_{Y}}^{F}\left(\left(L_{\alpha}, F\right),\left(K_{Y}[1], F\right)\right)=\operatorname{Ham}_{Y}\left(G r_{-p}^{F} L_{\alpha}, K_{Y}[1]\right)
\end{aligned}
$$

so that $\mathbb{D}(M, F)$ is well-defined, i.e. the filtered acyclic condition is preserved, where we use the filtration $G$ and (1.3.13). By a similar argument we check that the coherence condition (1.3.10) is preserved and $\mathcal{H}^{j} G r^{F} \mathbb{D}\left(M_{\bullet}, F\right)=0$ for $j \gg 0$. As to the condition (1.3.9), we use the canonical filtration $\tau$ (with the equivalence (1.3.15)) and the generalization of [S1, 2.1.16] to the case where the filtered Modules have the action of $N$, can, Var so that the assertion is reduced to the case where $N=0(-1<\alpha<0)$ and $M_{-1}$ or $M_{0}$ is zero (using some filtration on 
$\left.\mathscr{H}^{j}\left(\oplus_{p} F_{p} M\right)\right)$, then the assertion is clear. Thus we get the dual functor

$$
\mathbb{D}: D_{m c}^{b} G F\left(G r^{V} \mathscr{D}_{X}\right) \rightarrow D_{m c}^{b} G F\left(G r^{V} \mathscr{D}_{X}\right)^{\circ},
$$

using (1.3.15-16). By definition it induces

$$
\text { D: } D_{m c}^{b} G F\left(G r^{V} \mathscr{D}_{X}\right)^{(\alpha)} \rightarrow\left(D_{m c}^{b} G F\left(G r^{V} \mathscr{D}_{X}\right)^{(-1-\alpha)}\right)^{\circ},
$$

because the degree of $K_{Y}[1] \otimes 1$ is -1 and $(m \otimes P) t(s)=m \otimes(-s-1) P$ (where we use the canonical resolution in 1.4). Similarly we check that the dual functor $\boldsymbol{D}$ is compatible with the functors $\Psi^{\alpha}$, i.e.

$$
\begin{aligned}
& \Psi^{-1-\alpha} \mathbb{D}\left(M_{\bullet}, F\right)=\left(\mathbb{D} \Psi^{\alpha}\left(M_{\bullet}, F\right)\right)(-1) \text { for }-1<\alpha<0, \\
& \Psi^{-1} \mathbb{D}\left(M_{\bullet}, F\right)=\left(\mathbb{D} \Psi^{-1}\left(M_{\bullet}, F\right)\right)(-1), \\
& \Psi^{0} \mathbb{D}\left(M_{\bullet}, F\right)=\mathbb{D} \Psi^{0}\left(M_{\bullet}, F\right),
\end{aligned}
$$

so that the action of $N$, can, Var on the left hand side is identified with the transpose of $-N$, - Var, can on the right hand side respectively, because the morphisms $u^{\prime}$ and $u^{\prime \prime}$ in 1.4 are (essentially) self-dual. Here $(k)$ for an integer $k$ means the Tate twist so that $(M, F)(k)=(M, F[k])$ for a filtered Module $(M, F)$. As a corollary of (1.5.3), $M G F_{m h C M}\left(G r^{V} \mathscr{D}_{X}\right)$ is stable by the functor $\mathbb{D}$ and we get the functor

$$
D: M G F_{m h C M}\left(G r^{V} \mathscr{D}_{X}\right) \rightarrow M G F_{m h C M}\left(G r^{V} \mathscr{D}_{X}\right)^{\circ} .
$$

Let $\operatorname{MF}_{m h C M}\left(\mathscr{D}_{X}\right)$ be the full subcategory of $M F_{h C M}\left(\mathscr{D}_{X}\right)$ in 1.1 such that its objects have the filtration $V$ satisfying (1.2.1-7) and $\psi(M, F), \phi_{1}(M, F)$ are CohenMacaulay. Then we have the vanishing cycle functors

$$
\psi, \phi_{1}: M F_{m h C M}\left(\mathscr{D}_{X}\right) \rightarrow M F_{h C M}\left(\mathscr{D}_{Y}\right) .
$$

1.6. Theorem. The full subcategory $M F_{m h C M}\left(\mathscr{D}_{X}\right)$ of $M F_{h C M}\left(\mathscr{D}_{X}\right)$ is stable by the dual functor $\mathbb{D}$, and we have the functorial isomorphisms:

$$
\begin{aligned}
& \psi \boldsymbol{D}(M, F)=(\boldsymbol{D} \psi(M, F))(1) \\
& \phi_{1} \mathbb{D}(M, F)=\boldsymbol{D} \phi_{1}(M, F)
\end{aligned}
$$

such that the action of $s$ on $\psi_{\neq 1} D(M, F)$ is identified with the transpose of $-1-s$ on $\psi_{\neq 1}(M, F)\left(:=\bigoplus_{-1<\alpha<0} G r_{a}^{V}(M, F[1])\right)$, and can, Var on the left hand side with the transpose of -Var, can on the right.

Proof. It is enough to show the first assertion and the natural isomorphism:

$$
\mathcal{D} \operatorname{Gr}^{V}(M, F)=G r^{V} \mathbb{D}(M, F) \text { for }(M, F) \in M F_{m l l C M}\left(\mathscr{D}_{X}\right) \text {. }
$$


Locally we have a resolution $(M, F) \leftarrow(L, F)$ such that $\left(L^{i}, F\right)$ are finite direct sums of the copies of $\left(\mathscr{D}_{X} ; F[p], V[\alpha]\right)(-1 \leqq \alpha \leqq 0)$ and $G r^{V}(M, F) \leftarrow G r^{V}(L, F)$ is also a filtered quasi-isomorphism. Then for the calculation of $\boldsymbol{D}(M, F)$, (resp. $\boldsymbol{D} G r^{V}(M, F)$ ), we can use the above resolution and replace $K_{X}\left(\right.$ resp. $\left.K_{Y}[1]\right)$ by $\omega_{X}[n]$ (resp. $\left.\omega_{Y}[n]\right)$ in (1.1.2) (resp. (1.5.1)), and this complex is denoted by $\boldsymbol{D}(L, F)$ (resp. $\boldsymbol{D} G r^{V}(L, F)$ ). Then $\boldsymbol{D}(L, F)$ has the filtration $V$ by

$$
\boldsymbol{D}(L ; F, V)=\operatorname{Ham}_{\mathscr{D}_{X}}^{F}\left((L ; F, V),\left(\omega_{X}[n] ; F, V\right) \otimes \mathcal{O}_{X}\left(\mathscr{D}_{X} ; F, V\right)\right),
$$

where $\omega_{X}$ has the filtration $V$ as a right $\mathscr{D}_{X}$-Module such that $G r_{-i}^{V} \omega_{X}=\omega_{Y} \otimes$ $t^{i} d t / t$ for $i>0$ (and 0 otherwise). By definition of $\boldsymbol{D} G r^{V}(L, F)$ in 1.5 , we have the canonical isomorphism:

$$
G r^{V} \boldsymbol{D}(L, F)=\boldsymbol{D} G r^{V}(L, F)
$$

as complexes of filtered graded $G r^{V} \mathscr{D}_{X}$-Modules, because

$$
\operatorname{Gr}^{V}\left(\omega_{X} \otimes \mathcal{O}_{X} \mathscr{D}_{Y}\right)=\omega_{Y} \otimes \mathcal{O}_{Y} G r^{V} \mathscr{D}_{X}
$$

as right $G r^{V} \mathscr{D}_{X}$-bi-Modules. Then by [S1, 3.3.3-5] and (1.5.4) (cf. the proof of $[S 1,5.1 .13]), \boldsymbol{D}(L ; F, V)$ is bi-strict (cf. [S1, 1 2.2]), and by the above quasiisomorphism, the filtration $V$ on $\boldsymbol{D}(L, F)$ induces the filtration $V$ on $\boldsymbol{D}(M, F)$ satisfying (1.2.1-7). Thus we get the first assertion and (1.6.2) locally. We check that this isomorphism is independent of the local resolution $(L, F)$ and (1.6.2) is globally well-defined.

1.7. Remark. The arguments in this section can be applied to the non filtered case as follows. Let $X, Y$ be as in 1.2, and $M\left(\mathscr{D}_{X}\right)_{Y}$ the category of coherent $\mathscr{D}_{X}$-Modules having the filtration of Malgrange-Kashiwara along $Y$. Here we choose and fix an ordering of $\boldsymbol{C}$ such that $a<a+1, a+m<b$ for some $m \in \boldsymbol{Z}$, and $a<b \Leftrightarrow-b<-a \Leftrightarrow a+1<b+1$ for any $a, b \in C$. Put $\Lambda=\{a \in C:-1 \leq a \leq$ $0\}$, and $\Lambda^{\prime}=\Lambda \backslash\{0\}$. Then $M \in M\left(\mathscr{D}_{X}\right)_{Y}$ has the filtration $V$ indexed by $\boldsymbol{C}$ such that $V_{\alpha} M$ are coherent over $V_{0} \mathscr{D}_{X} D$ and $t \partial_{t}-\alpha$ is nilpotent on $G r_{\alpha}^{V} M$ (locally on $X$ ). We define

$$
\psi M=\oplus_{\alpha \in A^{\prime}} G r_{a}^{V} M, \quad \psi_{1} M=G r_{-1}^{V} M, \quad \phi_{1} M=G r_{0}^{V} M
$$

which are independent of the choice of the ordering. Here note that the holonomic $\mathscr{D}_{X}$-Modules belong to $M\left(\mathscr{D}_{X}\right)_{Y}$ by [K3] (combined with the argument as in $[S 1,3.2 .4])$. As in [K2] we consider $M \in M\left(\mathscr{D}_{X}\right)_{Y}$ of the form Coker $u$ with $u$ a filtered endomorphism of a direct sum of copies of $\left(\mathscr{D}_{X}, V[\alpha]\right)(-1 \leq$ $\alpha \leq 0$ ) such that $G r^{V} u$ is a diagonal matrix whose restriction to a copy of $G r^{V[\alpha]}$ 
$\mathscr{D}_{X}$ is the left multiplication by $\left(t \partial_{t}-\alpha\right)^{m}$ for $m \geq 0$ (but we do not assume that the off diagonal coefficients of the matrix belong to $V_{-1} \mathscr{D}_{X}$ ). Then choosing local generators of the filtration, every object of $M\left(\mathscr{D}_{X}\right)_{Y}$ has locally a surjective morphism from such a module (where $m$ may be 0 in the irregular case), and hence a resolution by such modules locally. This implies that for any filtered resolution of $(M, V)$ by finite direct sums of copies of $\left(\mathscr{D}_{X}, V[\alpha]\right)(-1 \leq \alpha \leq 0)$, its dual with value in $\left(\omega_{X} \otimes \mathscr{D}_{X}[n], V\right)$ is strict and its cohomologies with the induced filtration belong to $M\left(\mathscr{D}_{X}\right)_{Y}$, because it is clear for Coker $u$ as above by its self dual form and the injectivity of $G r^{V} u$, and we can apply the strictness of morphisms of $M\left(\mathscr{D}_{X}\right)_{Y}$, cf. [ $\left.\mathbb{K} 2\right][S 1,3.1 .6]$, by taking a free resolution of the above resolution, where we use also the independence of filtered free resolution, i.e. a complex of filtered finite free modules is filtered acyclic, iff it is acyclic and so is its graduation. Here the dual of a free graded module is defined by the (shifted) graded morphisms to $\omega_{Y} \otimes G r^{V} \mathscr{D}_{X}[n]$ as in 1.5 so that the dual functor $\mathbb{D}$ commutes with $G r^{V}$ for a complex of finite free filtered modules, and $\mathscr{H}^{j} \mathbb{D}$ commutes with $G r^{V}$ by the above argument, because the graduation commutes with $\mathscr{H}^{j}$ for a strict complex. Then we get the commutativity of $\psi_{2} \phi_{1}$ with $\mathcal{H}^{j} \mathbb{D}$ using the resolution 1.4 in the non filtered case, where we apply it to the standard resolution of $\psi M, \phi_{1} M$ by induced modules using the local nilpotency of the action of $N$ for its proof. We get also the correspondence between can, Var, $N$ on $\psi \mathcal{H}^{j} \mathbb{D} M, \phi_{1} \mathcal{H}^{j} \mathbb{D} M$ and the transpose of - Var, can, $-N$ on $\psi M$, $\phi_{1} M$.

1.8. Remark. Here we give some formalism of sign of complexes, cf. [D2], which will be needed in the proof of 2.2 .

Let $A, B$ be complexes (of abelian groups or sheaves). We define the complex ffom $(A, B)$ so that we have a canonical isomorphism (without sign):

$$
\operatorname{Hom}(Z, \operatorname{Hom}(A, B))=\operatorname{Hom}(Z \otimes A, B) \text { for any complex } Z \text {. }
$$

For $Z=\operatorname{Ham}(A, B)$, we have $Z^{i}=\mathbb{I I}_{j} \not \operatorname{tam}\left(A^{j}, B^{i+j}\right)$ with

$$
(d f)(x)=-(-1)^{i} f(d x)+d(f(x)) \text { for } f \in Z^{i}, x \in A^{j} .
$$

By the canonical isomorphism $Z[m] \otimes A[-m]=Z \otimes A$ for $m \in \mathbb{Z}$, we get

$$
\operatorname{Hom}(Z[m], \operatorname{Hom}(A[-m], B)=\operatorname{Hom}(Z \otimes A, B)
$$

and an isomorphism

$$
(\operatorname{Ham}(A, B))[m] \simeq \operatorname{Ham}(A[-m], B),
$$


where the sign $(-1)^{i m}$ appears on $\operatorname{Ham}\left(A^{j}, B^{i+j}\right)$ (here $(-1)^{(i+m) m}$ is also possible). Similarly we have

$$
(\operatorname{Ham}(A, B))[m] \simeq \operatorname{Ham}(A, B[m]),
$$

where no sign appears. The problem is that the isomorphism (1.8.3) is not compatible with composition for $m, n \in \mathbb{Z}$, and does not commute with the isomorphism (1.8.4). Let $f: A \rightarrow A^{\prime}$ be a morphism of complexes, and $f^{*}: Z^{\prime} \rightarrow$ $Z$ its transpose, where $Z^{\prime}=\operatorname{Ham}\left(A^{\prime}, B\right)$ and $\left\langle f^{*} v, u\right\rangle=\langle v, f u\rangle \in B^{i+j}$ for $u \in A^{i}$, $v \in \operatorname{Hom}\left(A^{\prime i}, B^{i+j}\right) \subset Z^{\prime i}$. Then by 3.6 and

$$
\begin{gathered}
\operatorname{Hom}\left(C\left(Z^{\prime} \stackrel{f^{*} \rightarrow}{\rightarrow} Z\right), \operatorname{Ham}\left(C\left(A \stackrel{f}{\rightarrow} A^{\prime}\right), B[1]\right)\right) \\
=\operatorname{Hom}\left(C\left(Z^{\prime} \stackrel{f^{*} \rightarrow}{\rightarrow}\right) \otimes C\left(A \stackrel{f}{\rightarrow} A^{\prime}\right), B[1]\right),
\end{gathered}
$$

we get an isomorphism

$$
C\left(\operatorname{Ham}\left(A^{\prime}, B\right) \stackrel{f^{*}}{\rightarrow} \operatorname{Ham}(A, B)\right)=\operatorname{Ham}\left(C\left(A \stackrel{f}{\rightarrow} A^{\prime}\right), B[1]\right)
$$

where the sign $(-1)^{i}$ appears on $\operatorname{Ham}\left(A^{j}, B^{i+j}\right)$.

\section{$\S 2$. Compatibility with the Topological Duality}

2.1. Topological duality for the vanishing cycle functors. With the notations of $\S 1$, let $\pi: \tilde{S}^{*} \rightarrow S^{*}$ be a universal covering, and we denote also by $\pi: \tilde{X}^{*} \rightarrow$ $X^{*}$ its cartesian product with $Y$, i.e. $\tilde{X}^{*}=Y \times \tilde{S}^{*}$, etc. Let $i: Y \rightarrow X$ and $\tilde{j}$ : $\tilde{X}^{*} \rightarrow X$ be the natural morphisms. For $K \in D_{c}^{b}\left(\boldsymbol{C}_{X}\right)$ we define $\psi K \in D_{c}^{b}\left(\boldsymbol{C}_{Y}\right)$ by

$$
\psi K=i^{*} \tilde{j}_{*} \tilde{j}^{*} K, \mathrm{cf} .[\mathrm{D} 1],
$$

where we may assume that $K$ is represented by a bounded complex of injective $\boldsymbol{C}_{X}$-Modules (i.e. flasque sheaves), and this representative is also denoted by $K$. The action of the monodromy $T$ is induced by the action of $r^{*}$ on $i^{*} \tilde{j}_{*} \tilde{j}^{*} K$, where $r$ is a generator of the covering transformation group of $\pi$ defined by $x \mapsto x+1$ if $\pi$ is identified with the exponential map $x \mapsto \exp (2 \pi i x)$. Then we have the natural decomposition (cf. [S1, 3.4.14]):

$$
\psi K=\oplus_{\lambda \in C^{*}} \psi_{\lambda} K,
$$

where $\psi_{\lambda} K$ is a subcomplex of $\psi K$ and the union of the kernel of $(T-\lambda)^{i}$ for $i \in N$. Put

$$
\psi_{\neq 1} K=\oplus_{\lambda \neq 1} \psi_{\lambda} K
$$


so that $\psi K=\psi_{1} K \oplus \psi_{\neq 1} K$. We define ${ }^{p} \psi K=\psi K[-1]$ (same for ${ }^{p} \psi_{1},{ }^{p} \psi_{\neq 1}$ ). Then we have the natural morphism

$$
\mathrm{sp}: i * K[-1] \rightarrow{ }^{p} \psi_{1} K\left(\text { or }{ }^{p} \psi K\right),
$$

and ${ }^{p} \phi_{1} K$ (or $\left.{ }^{p} \phi K\right)$ is defined by the mapping cone of sp. We have the natural morphism

$$
\text { can: }{ }^{p} \psi_{1} K \rightarrow{ }^{p} \phi_{1} K, \quad \text { can: }{ }^{p} \psi K \rightarrow{ }^{p} \phi K
$$

induced by the natural inclusion, and the decomposition ${ }^{p} \phi={ }^{p} \phi_{1} \oplus^{p} \phi_{\neq 1}$ compatible with that for ${ }^{p} \psi$ by the morphism can, where can induces an isomorphism ${ }^{p} \psi_{\neq 1} \underset{\rightarrow}{\rightarrow} \phi_{\neq 1}$. By definition can is imbedded into the distinguished triangle:

$$
\rightarrow{ }^{p} \psi_{1} K \stackrel{\text { can }}{\longrightarrow}{ }^{p} \phi_{1} K \rightarrow i^{*} K \stackrel{+1}{\longrightarrow}
$$

We define the action of $N$ on ${ }^{p} \psi_{\lambda} K,{ }^{p} \phi_{1} K$ by the logarithm of the unipotent part of the monodromy $T$, divided by $2 \pi i$, which is well-defined by definition of ${ }^{p} \psi_{\lambda} K$. We define the morphism Var: ${ }^{p} \phi_{1} K \rightarrow{ }^{p} \psi_{1} K$ by the cone of $(0, N)$. Here we omit the Tate twist (-1) using the canonical isomorphism $\mathbb{C}(-1)=$ $\mathbb{C}$, cf. [S1, (2.0.2)]. We have the natural quasi-isomorphism:

$$
i^{1} K \rightarrow\left[i^{*} K \stackrel{\mathrm{sp}}{\rightarrow} \psi_{1} K \stackrel{-N}{\longrightarrow} \psi_{1} K\right]
$$

where the right hand side is the single complex associated to the double complex such that $i^{*} K$ has the degree 0 with respect to the first index. From now on, $i^{!} K$ will denote the target of the quasi-isomorphism (2.1.4). Then the natural morphism $i^{l} K \rightarrow i^{*} K$ is identified with the projection onto the first component of $i^{!} K$. We define the morphism cosp: ${ }^{p} \psi_{1} K[-1] \rightarrow i^{!} K$ by the natural inclusion of $\psi_{1} K[-2]$ into the third component of $i ! K$. Then we have the natural quasi-isomorphism:

$$
C\left(\operatorname{cosp}:{ }^{p} \psi_{1} K[-1] \rightarrow i^{1} K\right) \rightarrow\left[i^{*} K \rightarrow \psi_{1} K\right]\left(={ }^{p} \phi_{1} K\right)
$$

induced by the natural projection. In particular we get the distinguished triangle:

$$
\rightarrow i^{1} K \rightarrow{ }^{p} \phi_{1} K \stackrel{\operatorname{Var}}{\longrightarrow}{ }^{p} \psi_{1} K \stackrel{+1}{\longrightarrow}
$$

because Var is homotopic to the natural projection of the mapping cone onto the first component.

Let $L$ be as $K$, and $\mathbb{S}: K \otimes L \rightarrow T_{X}$ a morphism of complexes, where $T_{X}$ is 
a flasque (i.e. $\boldsymbol{C}$-injective) resolution of $\boldsymbol{C}_{X}(n)[2 n]=\boldsymbol{C}_{X}[2 n]$. Then it induces $\psi S: \psi K \otimes \psi L \rightarrow \psi T_{X}, \quad \psi_{1} S: \psi_{1} K \otimes \psi_{1} L \rightarrow \psi_{1} T_{X}$.

We define ${ }^{p} \psi S:{ }^{p} \psi K \otimes \otimes^{p} \psi L \rightarrow \psi T_{X}[-2]$ so that the composition

$\psi K \otimes \psi L=\left(\boldsymbol{C}[1] \otimes^{p} \psi K\right) \otimes\left(\boldsymbol{C}[1] \otimes^{p} \psi L\right) \rightarrow(\boldsymbol{C}[1] \otimes \boldsymbol{C}[1]) \otimes\left({ }^{p} \psi K \otimes^{p} \psi L\right)$

$$
\stackrel{p}{\stackrel{p}{\longrightarrow} \mathbf{S}} \psi T_{X}
$$

coincides with $\psi \mathbf{S}$ (same for ${ }^{p} \psi_{1} \mathbf{S}$ ), where the second morphism is defined by

$$
x \otimes y \otimes z \otimes w \mapsto(-1)^{\operatorname{deg} y \cdot \operatorname{deg} z} x \otimes z \otimes y \otimes w .
$$

Here note that the natural morphisms

$$
i^{*} T_{X} \rightarrow \psi_{1} T_{X} \rightarrow \psi T_{X}, \quad \operatorname{cosp}: \psi_{1} T_{X}[-2] \rightarrow i^{1} T_{X}
$$

are quasi-isomorphisms. We denote also by ${ }^{p} \psi_{1} S$ its composition with $\psi_{1} T_{X}[-2] \rightarrow i^{!} T_{X}$. We have a pairing

$$
i^{!} \mathbf{S}: i^{*} K \otimes i^{!} L \rightarrow i^{!} T_{X}
$$

by $\left(i * \boldsymbol{S}, \psi_{1} \boldsymbol{S}, \psi_{1} \boldsymbol{S}\right)$ using the identification (2.1.4). Here we have a change of sign coming from the isomorphism

$$
i^{*} K \otimes\left(\psi_{1} L[-1]\right)=\left(i^{*} K \otimes \psi_{1} L\right)[-1]
$$

because [-1] means $\boldsymbol{C}[-1] \otimes_{\boldsymbol{C}}$ by definition. Then the sum of ${ }^{p} \psi_{1} \mathbf{S}$ and $i^{1} \mathbf{S}$ defines a pairing

$$
{ }^{p} \phi_{1} \mathbb{S}:{ }^{p} \phi_{1} K \otimes{ }^{p} \phi_{1} L \rightarrow i^{!} T_{X}
$$

and that of the distinguished triangles (2.1.3) and (2.1.5), cf. [S1, 5.2.3-4]. In particular we get

$$
{ }^{p} \psi_{1} \mathbf{S} \circ(\mathrm{id} \otimes \operatorname{Var})={ }^{p} \phi_{1} \mathbf{S} \circ(\mathrm{can} \otimes \mathrm{id}):{ }^{p} \psi_{1} K \otimes{ }^{p} \phi_{1} L \rightarrow i^{!} T_{X} .
$$

Here we can also use the pairing

$$
i^{!} S: i^{!} K \otimes i^{*} L \rightarrow i^{!} T_{X}
$$

similarly defined. Then the sum of $i^{!} \mathbf{S}$ and $-{ }^{p} \psi_{1} \mathbf{S}$ give a pairing ${ }^{p} \phi_{1} \mathbf{S}$ and that of (2.1.5) and (2.1.3) so that

$$
-{ }^{p} \psi_{1} S \circ(\operatorname{Var} \otimes \mathrm{id})={ }^{p} \phi_{1} \mathbf{S} \circ(\mathrm{id} \otimes \mathrm{can}):{ }^{p} \phi_{1} K \otimes{ }^{p} \psi_{1} L \rightarrow i^{!} T_{X} .
$$

We can check that these two definitions of ${ }^{p} \phi_{1} S$ are homotopic to each other, 
using the quasi-isomorphism

$$
\left[i^{*} K \rightarrow \psi_{1} K\right] \rightarrow C\left(\psi_{1} K[-2] \rightarrow\left[i^{*} K \rightarrow \psi_{1} K \rightarrow \psi_{1} K\right]\right)
$$

defined by $[x, y] \mapsto(N y,[x, y, 0])$. In fact the homotopy is given by

$$
\begin{gathered}
{\left[i^{*} K \rightarrow \psi_{1} K\right] \otimes\left[i^{*} L \rightarrow \psi_{1} L\right] \rightarrow\left[i^{*} T_{X} \rightarrow \psi_{1} T_{X} \rightarrow \psi_{1} T_{X}\right]} \\
\mathcal{U} \\
{[x, y] \otimes\left[x^{\prime}, y^{\prime}\right] \quad \mapsto \quad\left[0,{ }^{p} \psi_{1} \mathbb{S}\left(y, y^{\prime}\right), 0\right]}
\end{gathered}
$$

We say that a pairing $\mathbb{S}: K \otimes L \rightarrow T_{X}$ is non-degenerate, if the corresponding morphism:

$$
\mathbb{S}^{\mathfrak{R}}: K \rightarrow \mathbb{D} L=\operatorname{Ham}_{C}\left(L, T_{X}\right)
$$

is a quasi-isomorphism.

Remark. We can check that ${ }^{p} \psi \mathbb{S}$ and ${ }^{p} \phi_{1} \mathbb{S}$ are non-degenerate, if so is $\mathbb{S}$. In fact, we can reduce to the case where $K, L$ are perverse sheaves and then $K, L$ are simple (i.e. intersection complexes), using a filtration of $K, L$, because ${ }^{p} \psi$ and ${ }^{p} \phi_{1}$ are exact functors, cf. [BBD]. Then it is enough to prove the assertion for ${ }^{p} \psi S$ by (2.1.6) using the monodromy filtration of ${ }^{p} \psi_{1}$ and ${ }^{p} \phi_{1}$, cf. [S1, 5.1.12, 5.2.6]. The assertion follows from the same argument as in the proof of 3.5 , because

$$
\underset{\vec{n}}{\lim } \lim _{\leftarrow} i^{*} j_{*}\left(j^{*} L \otimes E_{n, m}\right)=C\left(N: \psi_{1} L \llbracket s \rrbracket\left[s^{-1}\right] \rightarrow \psi_{1} L\left[s \rrbracket\left[s^{-1}\right]\right)\right.
$$

is acyclic, where $E_{n, m}(n \geq m)$ is the pull-back of a local system on $S^{*}$ whose monodromy is unipotent and has one Jordan block of size $n-m+1$, and the natural injective and surjective morphisms $E_{n, m} \rightarrow E_{n+1, m}$ and $E_{n, m} \rightarrow E_{n, m+1}$ are given in a compatible way. (We can also use resolution of singularity and apply 3.5.)

2.2. Theorem。 For $(M, F) \in M F_{m h c M}\left(\mathscr{D}_{X}\right)$, put $L=D R_{X} M \in \operatorname{Perv}\left(\mathbb{C}_{X}\right), K=\mathbb{D} L$ so that we have the natural non-degenerate pairing $\mathbb{S}: K \otimes L \rightarrow T_{X}$. Then by the functorial isomorphisms $D R_{Y} \psi={ }^{p} \psi D R_{X}\left(\right.$ same for $\left.\phi_{1}\right), c f$. [S1, 3.4.12], and $D R \mathbb{D}$ $=\mathbb{D} D R, c f .[\mathrm{S} 1,2.4 .12]$, the duality isomorphism (1.6.1) corresponds to

$$
\begin{aligned}
& { }^{p} \psi \mathbb{S}^{*}:{ }^{p} \psi K \cong \mathbb{D}^{p} \psi L, \\
& { }^{p} \phi_{1} \mathbb{S}^{*}:{ }^{p} \phi_{1} K \underset{\sim}{\rightarrow} \mathbb{D}^{p} \phi_{1} L \text {. }
\end{aligned}
$$

Proof. The assertion is local. By restricting $X$, we have free resolutions:

$$
D R_{Y}^{-1}\left(L_{\alpha}, F\right) \rightarrow\left(G r_{\alpha}^{V} M, F\right) \text { for }-1 \leq \alpha \leq 0,
$$


where $\left(L_{\alpha}, F\right)$ are filtered differential complexes on $Y$ such that the filtration $F$ on each $L_{\alpha}^{j}$ splits and $G r_{p}^{F} L_{\alpha}^{j}$ are free $\mathcal{O}_{Y}$-Modules. Here $D R_{Y}^{-1}\left(L_{\alpha}, F\right)$ is the corresponding complex of filtered free $\mathscr{D}_{Y}$-Modules, cf. [S1, 2.2], and we may assume that $L_{\alpha}$ are bounded complexes and $F$ is finite, cf. [S1, 2.1.17]. Using a filtration of $G r_{\alpha}^{V} M$, we may choose the resolutions so that the morphisms can, Var, $N$ are lifted to $\left(L_{\alpha}, F\right)$ and $N$ is nilpotent. Then we get a free resolution of $\left(G r^{V} M, F\right)$ by 1.4 , and it can be lifted to a free resolution $D R_{X}^{-1}(L, F)$ of $(M, F)$ by restricting $X$. By definition $\left(L^{j}, F\right)$ is the direct sum of $\Theta_{X} \otimes \mathcal{O}_{Y}$ $\left(L_{\alpha}^{j}, F\right)(-1 \leqq \alpha \leqq 0)$ and $\mathcal{O}_{X} \otimes \mathcal{O}_{Y}\left(L_{\alpha}^{j+1}, F[1]\right)(-1 \leqq \alpha<0), \mathcal{O}_{X} \otimes \mathcal{O}_{Y}\left(L_{0}^{j+1}, F\right)$, and $L^{j}$ has the filtration $V$ such that $V_{\beta} L^{j}$ is the direct sum of $t^{i} \Theta_{X} \otimes L^{j}$ for $i=\max$ $(0,-[\beta-\alpha])$ and $t^{i} \Theta_{X} \otimes L^{j+1}$ for $i=\max (0,-[\beta-\alpha])(\alpha \neq-1,0)$, $\max (0$, $-[\beta+1])(\alpha=0), \max (0,-[\beta])(\alpha=-1)$. Note that $\operatorname{Gr}^{V} D R_{X}^{-1}(L, F)$ is the above resolution of $\left(G r^{V} M, F\right)$ and

$$
D R_{X}^{-1}(L ; F, V) \rightarrow(M ; F, V)
$$

is a bifiltered resolution on a neighborhood of $Y$, where

$$
V_{\alpha}\left(D R_{X}^{-1} L^{j}\right)=\sum_{\beta+\gamma=\alpha} V_{\beta} L^{j} \otimes V_{\gamma} \mathscr{D}_{X} .
$$

For $\alpha \in \mathbb{Q}$, let $\mathcal{O}_{X}^{\alpha}\left(\right.$ resp. $\left.\tilde{\mathcal{O}}_{X}^{\alpha}\right)$ be the left $\mathscr{D}_{X}$-sub-Module of $i_{*} i^{*} \tilde{j}_{*} \tilde{j}^{*} \mathcal{O}_{X}$ generated by $t^{\alpha+j}\left(\operatorname{resp} \cdot t^{\alpha+j}(\log i)^{i}(i \geqq 0)\right)$ for $j \in \mathbb{Z}$ over $\mathcal{O}_{X}$. We define the decreasing filtration $V$ on $\mathcal{O}_{X}^{\alpha}$ and $\tilde{\mathcal{O}}_{X}^{\alpha}$ by

$$
\begin{aligned}
& V^{\beta} \mathcal{O}_{X}^{\alpha}=\mathcal{O}_{X} t^{\alpha-[\alpha-\beta]} \\
& V^{\beta} \tilde{\mathcal{O}}_{X}^{\alpha}=\sum_{i \geqq 0} \mathcal{O}_{X} t^{\alpha-[\alpha-\beta]}(\log t)^{i} .
\end{aligned}
$$

Put

$$
\begin{aligned}
& \left(\widetilde{L}^{(\alpha)}, V\right)=(L, V) \otimes \Theta_{X}\left(\tilde{\Theta}_{X}^{\alpha}, V\right)\left(\subset i_{*} i^{*} \tilde{j}_{*} \tilde{j}^{*} L\right) . \\
& \left(\widetilde{L}^{(\alpha)}[s], V\right)=\left(D R_{S}^{-1} L, V\right) \otimes \Theta_{X}\left(\tilde{\mathcal{O}}_{X}^{\alpha}, V\right)\left(\subset i_{*} i^{*} \tilde{j}_{*} \tilde{j}^{*} D R_{S}^{-1} L\right)
\end{aligned}
$$

where $D R_{S}^{-1} L=D R_{Y} D R_{X}^{-1} L=L \otimes \mathcal{O}_{S} \mathscr{D}_{S}$ and

$$
V_{\alpha} D R_{S}^{-1} L^{j}=\sum_{\beta+\gamma=\alpha} V_{\beta} L^{j} \otimes \Theta_{S} V_{\gamma} \mathscr{D}_{S} .
$$

Then $\tilde{L}^{(\alpha)}$ is a subcomplex of $i_{*} i^{*} \tilde{j}_{*} \tilde{j}^{*} L$ (same for $\tilde{L}^{(\alpha)}[s]$ ), and $\tilde{L}^{(\alpha)}[s]$ is a complex of right $\mathscr{D}_{S}$-Modules such that

$$
0 \rightarrow V_{\beta} \tilde{L}^{(\alpha)}[s] \stackrel{s}{\rightarrow} V_{\beta} \tilde{L}^{(\alpha)}[s] \rightarrow V_{\beta} \tilde{L}^{(\alpha)} \rightarrow 0 .
$$

Then by [S1, 3.4.12] the natural morphisms

$$
\begin{aligned}
& G r_{0}^{V} \tilde{L}^{(\alpha)}[s] \leftarrow V_{0} \tilde{L}^{(\alpha)}[s] \rightarrow \tilde{L}^{(\alpha)}[s] \\
& G r_{0}^{V} \tilde{L}^{(\alpha)} \leftarrow V_{0} \tilde{L}^{(\alpha)} \rightarrow \widetilde{L}^{(\alpha)} \rightarrow \psi_{\exp (2 \pi i \alpha)} J L
\end{aligned}
$$


are quasi-isomorphisms for $-1<\alpha \leqq 0$, where $J L$ is the canonical flasque resolution (of Godement) of $L$.

We define the filtration $V$ on $\Omega_{X}(\log Y)$ by

$$
V^{\alpha} \Omega_{X}(\log Y)=t^{i} \Omega_{X}(\log Y) \text { for } i=\max (0,-[-\alpha]),
$$

and $V$ on $\Omega_{X}$ (resp. $\omega_{X}$ ) using the inclusion $\Omega_{X} \rightarrow \Omega_{X}(\log Y)$ (resp. the isomorphism $\Omega_{X}^{n}=\omega_{X}$ ). Then we can define $\tilde{\Omega}_{X}^{(\alpha)}, \tilde{\Omega}_{X}^{(\alpha)}[s]$ similarly (same for $\omega_{X}$ ). If $\alpha=0$, they are written also as $\widetilde{\Omega}_{X}, \widetilde{\Omega}_{X}[s]$.

Let $(D L ; F, V)$ be the dual filtered differential complex of $(L ; F, V)$, i.e.

$$
(\boldsymbol{D} L ; F, V)^{j}=\operatorname{Ham}_{O_{X}}^{F V}\left((L ; F, V)^{-j-n},\left(\omega_{X} ; F, V\right)\right),
$$

and sometimes $(\mathbb{D} L ; F, V)$ is denoted by $\operatorname{Ham}_{\mathcal{O}_{X}}^{F V}\left((L ; F, V),\left(\omega_{X} ; F, V\right)[n]\right)$. By definition we have the natural filtered quasi-isomorphism:

$$
\begin{aligned}
& \operatorname{Ham}_{\mathrm{Diff}_{X}}^{V}\left((L, V),\left(\Omega_{X}, V\right)[2 n]\right):=\operatorname{Ham}_{\mathscr{D}_{X}}^{V}\left(D R_{X}^{-1}(L, V), D R_{X}^{-1}\left(\Omega_{X}, V\right)[2 n]\right) \\
& \quad=D R_{X} D R_{X}^{-1}(D L, V) \rightarrow(\mathbb{D} L, V),
\end{aligned}
$$

because $\operatorname{Ham}_{\text {Diff }_{X}}^{V}\left((L, V),\left(\omega_{X}, V\right)[n]\right)=D R_{X}^{-1}(D L, V)$ cf. [S1, 2.2]. We can check that (2.2.4) induces a filtered quasi-isomorphism

$$
H_{\text {Diff }}\left((L, V),\left(\tilde{\Omega}_{X}^{(\alpha)}, V\right)[2 n]\right) \rightarrow\left((\mathbb{D} \tilde{L})^{(\alpha)}, V\right)
$$

for $-1<\alpha \leqq 0$. Tensoring $\mathcal{O}_{X}^{\beta}$ for $\beta=-1-\alpha$, we get also

$$
\begin{aligned}
& V_{0} \operatorname{Ham}_{\operatorname{Diff}_{X}}\left(L, \tilde{\Omega}_{X}^{(\alpha)}[2 n]\right) \rightarrow V_{0} \operatorname{Ham}_{\mathrm{Diff}_{X}}\left(\tilde{L}^{(\beta)}, \tilde{\Omega}_{X}^{(-1)}[2 n]\right) \\
& \quad \rightarrow \operatorname{Ham}_{\mathrm{Diff}_{X}}\left(G r_{0}^{V} \tilde{L}^{(\beta)}, G r_{0}^{V} \tilde{\Omega}_{X}^{(-1)}[2 n]\right)
\end{aligned}
$$

for $-1<\alpha<0$. Here we have a quasi-isomorphism

$$
G r_{0}^{V} \tilde{\Omega}_{X}^{(-1)}[2 n]=C\left(s: \Omega_{Y}[\tau] \rightarrow \Omega_{Y}[\tau]\right)[2 n-1] \rightarrow \Omega_{Y}[2 n]
$$

as filtered differential complexes on $Y$, where $\tau=\log t$ and $s \tau^{i}=i \tau^{i-1}$, i.e. [s, $\left.\tau\right]=$ 1 in $\widetilde{\mathscr{D}}_{X}$. Therefore by $(2.2 .3)$ and $(2.2 .5-6)$ we get a morphism in $D_{c}^{b}\left(C_{Y}\right)$ :

$$
\begin{aligned}
& \psi_{\exp (2 \pi i \alpha)} \mathbb{D} L \approx G r_{0}^{V}(\widetilde{D L})^{(\alpha)} \\
& \quad \rightarrow \operatorname{Ham}_{\mathrm{Diff}_{\bar{Y}}}\left(G r_{0}^{V} \tilde{L}^{(\boldsymbol{\beta})}, \Omega_{Y}[2 n]\right) \cong\left(\mathbb{D} \psi_{\exp (2 \pi i \beta)} L\right)[2] .
\end{aligned}
$$

We can check that (2.2.7) coincides with $\psi_{\neq 1} \mathbb{S}^{\#}$, and it is enough to check that (2.2.7) coincides with the analytic duality. In the level of complex, (2.2.7) is represented by

$$
\begin{aligned}
C & \left(G r_{0}^{V}(\widetilde{D} L)^{(\alpha)}[s] \stackrel{\cdot s}{\rightarrow} G r_{0}^{V}(\widetilde{D L})^{(\alpha)}[s]\right) \\
& \rightarrow \operatorname{Hom}_{\mathcal{O}_{Y}}\left(G r_{0}^{V} \widetilde{L}^{(\beta)}, C\left(\omega_{Y}[\tau] \stackrel{-s}{\longrightarrow} \omega_{Y}[\tau]\right)[n]\right)
\end{aligned}
$$




$$
\underset{\sim}{\rightarrow} \operatorname{Hom}_{O_{Y}}\left(G r_{0}^{V} \tilde{L}^{(\beta)}, \omega_{Y}[n+1]\right)
$$

Here we have

$$
\begin{aligned}
& G r_{0}^{V}(\widetilde{\boldsymbol{D} L})^{(\boldsymbol{\alpha})}[s]=C\left(\left(\boldsymbol{D} L_{\boldsymbol{\beta}}\right)[s, \tau] \rightarrow\left(\boldsymbol{D} L_{\boldsymbol{\beta}}\right)[s, \tau]\right) \\
& G r_{0}^{V} \tilde{L}^{(\boldsymbol{\beta})}=C\left(L_{\boldsymbol{\beta}}[\tau] \rightarrow L_{\boldsymbol{\beta}}[\tau]\right)
\end{aligned}
$$

with the morphisms defined by

$$
\begin{aligned}
& u \otimes P \mapsto N^{*} u \otimes P+u \otimes s P \\
& v \otimes Q \mapsto N v \otimes Q-v \otimes s Q
\end{aligned}
$$

for $u \in \boldsymbol{D} L_{\boldsymbol{\beta}}, v \in L_{\boldsymbol{\beta}}, P \in \boldsymbol{C}[s, \tau], Q \in \boldsymbol{C}[\tau]$, and $u \otimes s^{i} \tau^{j} \in\left(\boldsymbol{D} L_{\boldsymbol{\beta}}\right)[s, \tau]$ corresponds to

$$
L_{\beta}[\tau] \ni \nu \otimes \tau^{k} \mapsto\langle u, v\rangle \otimes \tau^{j}(-\operatorname{ad} s)^{i} \tau^{k} \in \omega_{Y}[\tau]
$$

up to sign (cf. 1.8), where $(\operatorname{ad} s) P=[s, P]$. We define a quasi-isomorphism

$$
L_{\beta}[1] \rightarrow C\left(L_{\beta}[\tau] \rightarrow L_{\beta}[\tau]\right)
$$

by $u \mapsto\left(\sum_{i \geqq 0} N^{i} u \otimes \tau^{i} / i\right.$ !, 0). Then this represents the isomorphism in [S1, 3.4.12] (using (2.2.3)), because it can be lifted to a quasi-isomorphism:

$$
L_{\beta}[1] \ni v \mapsto\left[\begin{array}{cc}
\sum N^{i} v \otimes \tau^{i} / i !, & 0 \\
0 & , \sum N^{i} v \otimes \tau^{i} / i !
\end{array}\right] \in C\left[\begin{array}{cc}
L_{\beta}[s, \tau] \rightarrow L_{\beta}[s, \tau] \\
\uparrow \cdot s & \uparrow \cdot s \\
L_{\beta}[s, \tau] \rightarrow L_{\beta}[s, \tau]
\end{array}\right] .
$$

We apply this also to $\boldsymbol{D} L_{\beta}$ and compose these with (2.2.8) so that we get a morphism

$$
\left(\boldsymbol{D} L_{\beta}\right)[1] \rightarrow \operatorname{Hom}_{\mathcal{O}_{Y}}\left(L_{\beta}[1], \omega_{Y}[n-1]\right)[2]
$$

whose restriction to $\left(\boldsymbol{D} L_{\boldsymbol{\beta}}\right)^{i+1}$ is the identity multiplied by $(-1)^{i}$. This proves the assertion for $\psi_{\neq 1}$. For $\psi_{1}$, we use the quasi-isomorphisms (dual and quasi-inverse of each other):

$$
\begin{aligned}
G r_{0}^{V} \tilde{L}= & C\left(L_{0}[\tau] \oplus L_{-1}[\tau] \rightarrow L_{-1}[\tau] \oplus L_{0}[\tau]\right) \\
& \longleftrightarrow C\left(L_{-1}[\tau] \stackrel{N-S}{\longrightarrow} L_{-1}[\tau]\right)
\end{aligned}
$$

defined by

$$
\begin{aligned}
& \left(u \otimes P, v \otimes Q ; v^{\prime} \otimes Q^{\prime}, u^{\prime} \otimes P^{\prime}\right) \mapsto\left(v \otimes Q, v^{\prime} \otimes Q^{\prime}+\operatorname{Var} u^{\prime} \otimes P^{\prime}\right) \\
& (\operatorname{can} u \otimes P, u \otimes P ; v \otimes Q, 0) \hookrightarrow(u \otimes P, v \otimes Q)
\end{aligned}
$$

(same for $G r_{0}^{V} \tilde{L}[s]$ ). Then we can apply the same argument as above. As for 
$\phi_{1}$, we must be careful about the problem of homotopy and mapping cone. We use the diagram

$$
\begin{aligned}
& G r_{0}^{V} \operatorname{Ham}_{\text {Diff }_{X}}\left(L, \tilde{\Omega}_{X}[2 n]\right) \rightarrow \operatorname{Ham}_{\text {Diff }_{Y}}\left(G r_{0}^{V} \tilde{\mathbb{L}}, G r_{0}^{V} \tilde{\Omega}_{X}[2 n]\right) \\
& \uparrow \quad \| \\
& i^{*} V_{0} \operatorname{Ham}_{\mathrm{Diff}_{X}}\left(L, \tilde{\Omega}_{X}[2 n]\right) \rightarrow \operatorname{Ham}_{\mathrm{Diff}_{Y}}\left(G r_{0}^{V} \tilde{L}, G r_{0}^{V} \widetilde{\Omega}_{X}[2 n]\right) \\
& \downarrow \\
& i^{*} \operatorname{Ham}_{\mathrm{Diff}_{X}}\left(L, \widetilde{\Omega}_{X}[2 n]\right) \rightarrow \operatorname{Ham}_{C}\left(V_{0} \tilde{L}, J\left(\tilde{\Omega}_{X} / V_{<0} \tilde{\Omega}_{X}\right)[2 n]\right) \\
& \| \\
& i^{*} \operatorname{Hom}_{\mathrm{Diff}_{X}}\left(L, \tilde{\Omega}_{X}[2 n]\right) \rightarrow \operatorname{Ham}_{\mathbb{C}}\left(\tilde{L}, J \tilde{\Omega}_{X}[2 n]\right) \\
& i^{*} \operatorname{Ham}_{\mathbb{C}}\left(L, \tilde{j} \tilde{j}_{*} \stackrel{\downarrow j}{*} D R\left(K_{X}\right)\right)_{1} \rightarrow \operatorname{Ham}_{\mathbb{C}}\left(\tilde{L}, J \psi_{1} D R\left(K_{X}\right)\right) \\
& \psi_{1} \operatorname{Hom}_{\mathbb{C}}\left(L, D R\left(K_{X}\right)\right) \rightarrow \operatorname{Hom}_{\mathbb{C}}\left(\psi_{1} L, \psi_{1} D R\left(K_{X}\right)\right)
\end{aligned}
$$

and the similar two diagrams where $\tilde{L}, \psi_{1} L$ on the right hand side are replaced by $i * L$, and then $\tilde{\Omega}_{X}$ and $\psi_{1}$ are further replaced by $\Omega_{X}$ and $i^{*}$ and $\tilde{j}_{*} \tilde{j}^{*}, 1$ are omitted. Here $J$ means the canonical flasque resolution. Then the morphism in $D_{c}^{b}\left(\mathbb{C}_{Y}\right)$ (cf. 2.1):

$$
\begin{aligned}
{ }^{p} \phi_{1} \mathbb{S}^{*}:\left[i * \mathbb{D} L \rightarrow \psi_{1} \mathbb{D} L\right] \rightarrow\left[i^{*} \mathbb{D} L \rightarrow \psi_{1} \mathbb{D} L \stackrel{-N}{\rightarrow} \psi_{1} \mathbb{D} L \stackrel{\mathrm{id}}{\leftarrow} \psi_{1} \mathbb{D D L}\right] \\
\quad \rightarrow \operatorname{Ham}\left(\left[i^{*} L \rightarrow \psi_{1} L\right],\left[i * T_{X} \rightarrow \psi_{1} T_{X} \stackrel{-N}{\rightarrow} \psi_{1} T_{X}\right]\right) \\
\quad \rightarrow \operatorname{Ham}\left(\left[i^{*} L \rightarrow \psi_{1} L\right], T_{Y}\right)
\end{aligned}
$$

is represented by

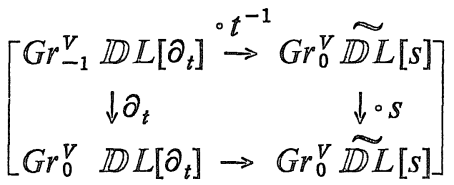

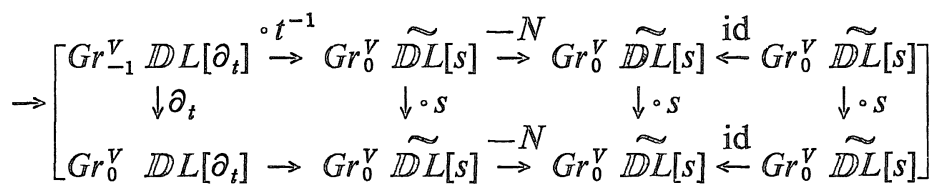

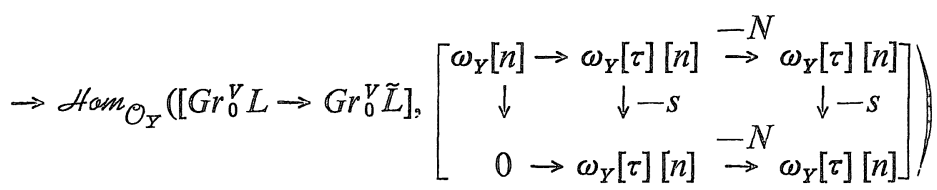

$$
\begin{aligned}
& \rightarrow \operatorname{Ham}_{O_{Y}}\left(\left[G r_{0}^{V} L \rightarrow G r_{0}^{V} \tilde{L}\right], \omega_{Y}[n-1]\right)
\end{aligned}
$$

where $[A \rightarrow B \rightarrow C \leftarrow D]$ means the mapping cone of $D[-2] \rightarrow[A \rightarrow B \rightarrow C]$, and 
the third morphism is defined by

$$
\left[\begin{array}{l}
a, b, c \\
0, e, f
\end{array}\right] \mapsto \operatorname{pr}(c+e)
$$

with pr the natural projection: $\omega_{Y}[\tau] \rightarrow \omega_{Y}$. Using the natural inclusion

$$
t^{-1}: G r_{-1}^{V} \boldsymbol{D} L\left[\partial_{t}\right]\left(=G r_{-1}^{V} D R_{S}^{-1} \boldsymbol{D} L\right) \rightarrow G r_{0}^{V} \boldsymbol{D} L\left[t^{-1}, s\right]
$$

and putting $\boldsymbol{D} L_{a}^{s}=\left(\boldsymbol{D} L_{a}\right)[s], \mathbb{D} L_{a}^{s \tau}=\left(\boldsymbol{D} L_{a}\right)[s, \tau]$, etc. for $a=-1,0$, the first diagram is expressed as

$$
\begin{gathered}
C\left(\boldsymbol{D} L_{0}^{s} \oplus \boldsymbol{D} L_{-1}^{s} \rightarrow \boldsymbol{D} L_{-1}^{s} \oplus \boldsymbol{D} L_{0}^{s}\right) \stackrel{(-\iota, \iota)}{\longrightarrow} C\left(\boldsymbol{D} L_{0}^{s \tau} \oplus \boldsymbol{D} L_{-1}^{s \tau} \rightarrow \boldsymbol{D} L_{-1}^{s \tau} \oplus \boldsymbol{D} L_{0}^{s \tau}\right) \\
\downarrow \cdot(-s, s) \\
C\left(\left(\boldsymbol{D} L_{0}^{s}\right) s \oplus \boldsymbol{D} L_{-1}^{s} \rightarrow\left(\boldsymbol{D} L_{-1}^{s}\right) s \oplus \boldsymbol{D} L_{0}^{s}\right) \stackrel{(-\iota, \iota)}{\longrightarrow} C\left(\boldsymbol{D} L_{0}^{s \tau} \oplus \boldsymbol{D} L_{-1}^{s^{\tau}} \rightarrow \boldsymbol{D} L_{-1}^{s^{\tau}} \oplus \boldsymbol{D} L_{0}^{s \tau}\right)
\end{gathered}
$$

with $c$ the natural inclusions, and into its associated single complex, we have a quasi-isomorphism of $\mathbb{D} L_{0}$ defined by

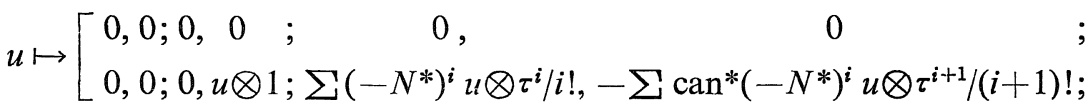

$$
\begin{aligned}
& -\Sigma \operatorname{can}^{*}\left(-N^{*}\right)^{i} u \otimes \tau^{i+1} /(i+1) !, 0 \\
& 0 \\
& , 0
\end{aligned}
$$

Here the bidegree of $G r_{0}^{V} \boldsymbol{D} L\left[\partial_{t}\right]$ in the first diagram is $(0,0)$. Similarly we have a quasi-isomorphism of $L_{0}$ into

$$
\left[G r_{0}^{V} L \rightarrow G r_{0}^{V} \tilde{L}\right]=\left[C\left(L_{-1} \rightarrow L_{0}\right) \stackrel{(-\iota, \iota)}{\longrightarrow} C\left(L_{0}^{\tau} \oplus L_{-1}^{\tau} \rightarrow L_{-1}^{\tau} \oplus L_{0}^{\tau}\right)\right]
$$

defined by

$$
v \mapsto\left[0, v ; \sum N^{i} v \otimes \tau^{i} / i !, \sum \operatorname{Var} N^{i} v \otimes \tau^{i+1} /(i+1) ! ; 0,0\right] .
$$

The composition of these morphisms with the above morphism representing ${ }^{p} \phi_{1} \boldsymbol{S}^{\sharp}$ coincides with the natural isomorphism

$$
\boldsymbol{D} L_{0} \rightarrow \text { Ham }_{O_{Y}}\left(L_{0}, \omega_{Y}[n-1]\right)
$$

where the contribution coming from $G r_{0}^{V} \widetilde{\boldsymbol{D}} L[s] \rightarrow \operatorname{Ham}\left(G r_{0}^{V} \tilde{L}, \omega_{Y}[\tau][n]\right)$ vanishes. This completes the proof of 2.2 .

2.3. Remark. Corresponding to the operation $L \mapsto \tilde{L}^{(\alpha)}$ in the proof of Theorem 2.2, we have the following for a complex of $\boldsymbol{C}$-Modules.

For $\alpha \in \boldsymbol{C} / \boldsymbol{Z}$, we define $E_{k}^{(\alpha)}$ to be the subsheaf of $\pi_{*} \boldsymbol{C}_{\tilde{s}^{*}}$ annihilated by $(T-\exp (2 \pi i \alpha))^{k+1}$ for $k \geqq 0$, where $T=r^{*}$ with $r$ the generator of the covering 
transformation as in 2.1. Then $E_{k}^{(\alpha)}$ is a local system on $S^{*}$, whose monodromy is given by $T^{-1}$. Put $E^{(\alpha)}=E_{\infty}^{(\alpha)}=\lim E_{k}^{(\alpha)} \subset \pi_{*} \mathbb{C}_{\widetilde{S}^{*}}$, and ${ }^{\infty} E_{k}^{(\alpha)}=\Gamma\left(\tilde{S}^{*}, \pi^{*} E_{k}^{(\alpha)}\right)$ (same for $\left.{ }^{\infty} E^{(\alpha)}\right)$. Let $t$ be the coordinate of $S$, and $z=(2 \pi i)^{-1} \log t$ that of $\tilde{S}^{*}$ so that $\gamma$ is given by $z \mapsto z+1$. We choose base points $p^{\prime}$ of $\tilde{S}^{*}$ and $p$ of $S^{*}$ so that $\pi\left(p^{\prime}\right)=p$ and $\operatorname{Re} z=0$ at $p^{\prime}$. Then $\pi^{-1}(p)=p^{\prime}+\mathbb{Z}$, and we have canonical isomorphisms:

$$
\Gamma\left(\tilde{S}^{*}, \pi^{*} \pi_{*} C_{\widetilde{s}^{*}}\right)=\left(\pi^{*} \pi_{*} \mathbb{C}_{\left.\widetilde{s}^{*}\right)_{p^{\prime}}}=\left(\pi_{*} \mathbb{C}_{\left.\widetilde{s}^{*}\right)_{p}}=\prod_{x \in p^{\prime}+Z} C_{x}\right.\right.
$$

so that the action of $T$ is induced by the canonical isomorphism $\mathbb{C}_{x+1} \rightarrow \mathbb{C}_{x}$, i.e. $\left(b_{x}\right)=T\left(a_{x}\right)$ satisfies $b_{x}=a_{x+1}$ for $a_{x}, b_{x} \in \mathbb{C}_{x}$. Therefore $E_{k}^{(\alpha)}$ is a local system of rank $k+1$, because its local section is uniquely determined by the values at $x=p^{\prime}+j(0 \leqq j \leqq k)$ using (2.3.1). Let $N$ be the logarithm of $T_{u}$, divided by $2 \pi i$, where $T=T_{u} T_{s}$ is the Jordan decomposition of $T$. Let $\tau$ be the element of ${ }^{\infty} E_{1}^{(0)}$ corresponding to $(2 \pi i k)_{x=p^{\prime}+k}$ by $(2.3 .1)$, and $e_{\alpha} \in E_{0}^{(\alpha)}$ to $(\exp (2 \pi i \alpha k))_{x=p^{\prime}+k}$. Then $N \tau=1$ and $T e_{\alpha}=\exp (2 \pi i \alpha) e_{\alpha}$.

We have a natural multiplication

$$
m: \pi_{*} \boldsymbol{C}_{\tilde{s}^{*}} \otimes \pi_{*} \mathbb{C}_{\tilde{s}^{*}} \rightarrow \pi_{*} \mathbb{C}_{\widetilde{s}^{*}}
$$

which induces the componentwise multiplication on $\mathbb{C}_{x}$ by (2.3.1). Then we have $T(m(u \otimes v))=m(T u \otimes T v)$ and

$$
\begin{aligned}
& m: E^{(\alpha)} \otimes E^{(\beta)} \rightarrow E^{(\alpha+\beta)}, \\
& m:{ }^{\infty} E^{(\alpha)} \otimes{ }^{\infty} E^{(\alpha)} \rightarrow{ }^{\infty} E^{(\alpha+\beta)}
\end{aligned}
$$

with $N(m(u \otimes v))=m(N u \otimes v)+m(u \otimes N v)$. In particular, we get

$$
{ }^{\infty} E^{(0)}=\mathbb{C}[\tau], \quad{ }^{\infty} E^{(\alpha)}=\mathbb{C}[\tau] e_{\sigma}
$$

as a $\mathbb{C}[N]$-algebra and a left $W$-Module, where $N \tau^{i}=i \tau^{i-1}$ and $W$ is the Weyl algebra $\mathbb{C}[s, \tau]$ such that $[s, \tau]=1$ and $s=N$.

Now we consider a left $\mathscr{D}_{S^{*}-\text { Module }} \pi_{*} \mathcal{O}_{\tilde{s}^{*}}$ or a left $\mathscr{D}_{S^{-M}}$ Module $j_{*} \pi_{*} \mathcal{O}_{\tilde{s}^{*}}$ with $j: S^{*} \rightarrow S$. Then $E_{k}^{(\alpha)}$ generates over $\mathcal{O}_{S^{*}}$ a $\mathscr{D}_{S^{*} \text {-sub-Module, and we denote }}$ by $\tilde{\mathcal{O}}_{k}^{\alpha}\left(\subset j_{*} \pi_{*} \mathcal{O}_{\widetilde{s}^{*}}\right)$ its natural extension as a meromorphic regular holonomic $\mathscr{D}_{s}$-Module (i.e. regular singular meromorphic connection). Then for each $\alpha \in \mathbb{C}$, we have a natural morphism

$$
{ }^{\infty} E_{k}^{(\alpha)} \ni u \mapsto \tilde{u}:=t^{\alpha} \exp (N \log t) u \in \Gamma\left(S, \tilde{\mathcal{O}}_{k}^{\alpha}\right)
$$

because $t^{\alpha} \exp (N \log t) u$ defines a section of $\pi^{*} \pi_{*} \mathcal{O}_{\tilde{s}^{*}}$ invariant by $T$. Then $\tilde{\tau}($ for $\alpha=0), \tilde{e}_{a} \in \Gamma\left(S^{*}, \pi_{*} \mathcal{O}_{\tilde{s}^{*}}\right)$ coincide with $\log t=2 \pi i z, t^{\alpha}=\exp (2 \pi i \alpha z) \in$ $\Gamma\left(\tilde{S}^{*}, \mathcal{O}_{\tilde{S}^{*}}\right)$ by definition. 
For $K \in D_{c}^{b}\left(\boldsymbol{C}_{X}\right)$ represented by an injective complex, we define

$$
\widetilde{K}^{(\alpha)}=i^{*} j_{*}\left(j^{*} K \otimes_{C} E^{(\alpha)}\right)=\lim _{\rightarrow} i^{*} j_{*}\left(j^{*} K \otimes_{C} E_{k}^{(\alpha)}\right) .
$$

Then $\widetilde{K}^{(\alpha)}$ is naturally quasi-isomorphic to (the underlying $\boldsymbol{C}$-complex of) $\tilde{L}^{(\alpha)}$, if $K$ is a flasque resolution of a filtered differential complex $L$ as in the proof of 2.2. Moreover the natural morphism

$$
\tilde{K}^{(\alpha)} \rightarrow \psi_{\exp (2 \pi i \alpha)} K
$$

is an isomorphism of complexes. In fact, by definition, $\psi_{\lambda} K$ is the inductive limit of

$$
i^{*} j_{*}\left(\operatorname{Ker}\left((T-\lambda)^{k}: \pi_{*} \pi^{*} K^{\prime} \rightarrow \pi_{*} \pi^{*} K^{\prime}\right)\right)
$$

where $K^{\prime}$ is the restriction of $K$ to $X^{*}$, and the natural morphism

$$
K^{\prime} \otimes_{C} E_{k}^{(\alpha)} \rightarrow \operatorname{Ker}\left((T-\lambda)^{k+1}: \pi_{*} \pi^{*} K^{\prime} \rightarrow \pi_{*} \pi^{*} K^{\prime}\right)
$$

is an isomorphism, because they are both subsheaves of $\pi_{*} \pi^{*} K^{\prime}$ and their local sections are uniquely determined by their values on the image of $k$ local sections of $\pi$. Here note that the monodromy of $\psi_{\exp (2 \pi i \alpha)} K$ corresponds to the action on $\tilde{K}^{(\alpha)}$ induced by the inverse of the monodromy of $E^{(\alpha)}$ (as a limit of local systems). The above construction can be generalized to the case where $K$ is defined on any field (cf. [S1, 3.4.14]).

2.4. Remark. The arguments in this section can be applied to the non filtered case as follows. With the notation of 1.7 we say that $M \in M\left(\mathscr{D}_{X}\right)_{Y}$ is cohomologically regular along $Y$, if the following conditions are satisfied:

$$
\begin{aligned}
& D R(M(* Y))=j_{*} j^{*} D R(M) \\
& D R(M(! Y))=j_{!} j^{*} D R(M)
\end{aligned}
$$

where $M(* Y)=M\left[1^{-1}\right]$ is the localization of $M$ by $t$, and $M(! Y)$ is the unique coherent $\mathscr{D}_{X}$-Module which is endowed with a morphism $M(! Y) \rightarrow M$ inducing an isomorphism outside $Y$, and satisfies $\partial_{t}: G r_{-1}^{V} M(! Y) \stackrel{\rightarrow}{\rightarrow} G r_{0}^{V} M(! Y)$, where $M(* Y)$ and $M(! Y)$ belong to $M\left(\mathscr{D}_{X}\right)_{Y}$ by the natural morphisms inducing isomorphisms outside $Y$. Note that (2.4.2) is equivalent to

(2.4.3) $D R_{Y}\left(\left.C\left(\partial_{t}: V_{\alpha-1} M \rightarrow V_{\alpha} M\right)\right|_{Y}\right)$ is acyclic for some (or any) $\alpha<0$

by [S1, 3.1.7], and in the holonomic case (2.4.2) is equivalent to (2.4.1) for the dual of $M$ by duality, cf. 1.7. We can show the commutativity of $\psi_{1}, \phi_{1}$ with $D R$ as in [S1, 3.4], if the following conditions are satisfied: 
(2.4.4) $M$ is cohomologically regular along $Y$,

(2.4.5) the action of the monodromy $T$ on each stalk of $\mathscr{H}^{k} \psi D R(M)$ has non zero minimal polynomial.

Here note that (2.4.5) is satisfied if $M$ is holonomic, and for the non unipotent monodromy part we can use the correspondence of the functor $M \mapsto j^{*} M \otimes_{\mathcal{O}} \tilde{\mathcal{O}}_{0}^{a s}$ with $K \mapsto j^{*} K \otimes E_{0}^{(\alpha)}$ by $D R$, cf. 2.3 for notation. In particular we have the commutativity of $\psi$ and $D R$, if $M$ satisfies (2.4.5) and $M \otimes \tilde{\mathcal{O}}_{0}^{\alpha}$ satisfies (2.4.4) for any $\alpha$ (e.g. $M$ is regular holonomic). The construction in 2.3 applies under the assumption (2.4.5), and the above assertion follows easily from (2.4.1) (2.4.3). In fact we have the isomorphisms as in [loc. cit]:

$$
\begin{aligned}
\psi_{1} & \left.D R(M) \approx \lim _{\overrightarrow{1}} i^{*} j_{*}\left(j^{*} D R(M) \otimes E_{k}^{(0)}\right)\right) \\
& \simeq \lim ^{*} D R\left(M(* Y) \otimes \tilde{\mathcal{O}}_{k}^{0}\right)=i^{*} D R(M(* Y)[\log t]) \\
& \simeq \overrightarrow{D R}_{Y}\left(\left.C\left(\partial_{t}: M(* Y)[\log t] \rightarrow M(* Y)[\log t]\right)\right|_{Y}\right) \\
& \leftarrow D R_{Y}\left(\left.C\left(\partial_{t} t: V_{-1} M[\log t] \rightarrow V_{-1} M[\log t]\right)\right|_{Y}\right) \\
& \simeq D R_{Y}\left(C\left(\partial_{t} t: G r_{-1}^{V} M[\log t] \rightarrow G r_{-1}^{V} M[\log t]\right)\right) \\
& \sim D R_{Y} G r_{-1}^{V} M
\end{aligned}
$$

where we use the natural filtration of $M(* Y) \otimes \tilde{\mathcal{O}}_{k}^{0}$ whose graduation is $M(* Y)$ for the second isomorphism. (A similar argument has been obtained by Mebkhout in the regular holonomic case using the expression of the finite determination vanishing cycles as limit of mapping cones due to Beilinson and the surjectivity of the variation on the vanishing cycles of holomorphic functions instead of the construction in 2.3.) Then in the regular holonomic case we get the compatibility of the dualities of $\mathscr{D}$-Modules and perverse sheaves for the vanishing cycle functors as in (2.2.1) by the same argument.

\section{§3. Normal Crossing Case}

3.1. Perverse sheaves with normal crossing supports. Let $X$ be a polydisc with the coordinates $\left(x_{1}, \cdots, x_{n}\right)$. Put $\bar{n}=\{1, \cdots, n\}$, and $\bar{\nu}=\left\{i \in \bar{n}: \nu_{i} \neq 0\right\}$ for $\nu=$ $\left(\nu_{1}, \cdots, \nu_{n}\right) \in(\mathbb{C} / \mathbb{Z})^{n}$. Let $\operatorname{Perv}\left(\boldsymbol{C}_{X}\right)_{n c}$ be the full subcategory of $\operatorname{Perv}\left(\mathbb{C}_{X}\right)$ such that the stratifications of its objects are given by the intersections of $D_{i}=\left\{x_{i}=0\right\}$. For $K \in \operatorname{Perv}\left(\boldsymbol{C}_{X}\right)_{n c}$, and $\nu \in(\mathbb{C} / \mathbb{Z})^{n}, I \subset \bar{n} \backslash \bar{\nu}$, we define

$$
\Psi_{1}^{\nu} K=\Psi_{1}^{\nu_{1}} \ldots \Psi_{n}^{\nu_{n}} K
$$

with $\Psi_{j}^{\nu_{j}}=\psi_{x_{j}, \exp \left(2 \pi i \nu_{j}\right)}$ (for $\left.j \notin I\right), \phi_{x_{j}, 1}$ (otherwise). Then we have the mor- 
phisms

$$
\begin{aligned}
& N_{i}: \Psi_{I}^{\nu} K \rightarrow \Psi_{I}^{\nu} K, \\
& \operatorname{can}_{i}: \Psi_{I}^{\nu} K \rightarrow \Psi_{I \cup\{i\}}^{\nu} K \text { for } i \notin I \cup \bar{\nu}, \\
& \operatorname{Var}_{i}: \Psi_{I}^{\nu} K \rightarrow \Psi_{I \backslash\{i\}}^{\nu} K \text { for } i \in I .
\end{aligned}
$$

We can check easily that $\Psi_{I}^{\nu}$ is independent of the order of $\bar{n}$, because $\psi_{x_{j}}$ is same as restricting to $\left\{x_{j}=1\right\}$ in this case. By Deligne's description of monodromical perverse sheaves, $K \in \operatorname{Perv}\left(\boldsymbol{C}_{X}\right)_{n c}$ is uniquely determined by $\Psi_{I}^{\nu} K$ with $N_{i}, \operatorname{can}_{i}, \operatorname{Var}_{i}$.

For $m=\left(m_{1}, \cdots, m_{n}\right) \in \boldsymbol{N}^{n}$, put $g=x^{m}=\prod x_{i_{i}}^{m_{i}}$, and $\bar{m}=\left\{i \in \bar{n}: m_{i} \neq 0\right\}$. The proof of the following lemma is pointed out by Kashiwara in the unipotent and reduced case (i.e. $\Psi_{I}^{\nu} K=0$ for $\nu \neq 0$, and $m_{i}=1$ ).

3.2. Lemma. For $\alpha \in \boldsymbol{C} / \boldsymbol{Z}, \nu \in(\boldsymbol{C} / \boldsymbol{Z})^{n}$ and $I \subset \bar{n} \backslash \bar{\nu}$, put $\left(N_{*}-m_{*} N\right)_{I \cap \bar{m}}=$ $\Pi_{i \in I \cap \bar{m}}\left(N_{i}-m_{i} N\right)$. Then for $K \in \operatorname{Perv}\left(\boldsymbol{C}_{X}\right)_{n c}$, we have a functorial isomorphism as $\boldsymbol{C}[N]-$ modules:

$$
\Psi_{I}^{\nu p} \psi_{g, \exp (2 \pi i \alpha)} K=\operatorname{Ker}\left(\left(N_{*}-m_{*} N\right)_{I \cap \bar{m}}: \Psi_{I \backslash \bar{m}}^{\nu+\alpha^{\prime} m} K \otimes_{\boldsymbol{C}} \boldsymbol{C}[\tau] \rightarrow \Psi_{I \backslash \bar{m}}^{\nu+\alpha^{\prime} m} K \otimes_{\boldsymbol{C}} \boldsymbol{C}[\tau]\right)
$$

such that $N_{i}, \operatorname{can}_{i}, \operatorname{Var}_{i}$ on the left hand side correspond respectively to the morphisms defined by the mapping cone of

$$
\begin{aligned}
& \left(N_{i}-m_{i} N, N_{i}-m_{i} N\right), \\
& \left(\mathrm{id}, N_{i}-m_{i} N\right) \quad \text { if } \quad i \in \bar{m} \backslash(I \cup \bar{\nu}),\left(\operatorname{can}_{i}, \operatorname{can}_{i}\right) \quad \text { if } i \notin \bar{m} \cup I \cup \bar{\nu}, \\
& \left(N_{i},-m_{i} N, \text { id }\right) \quad \text { if } \quad i \in I \cap \bar{m}, \quad\left(\operatorname{Var}_{i}, \operatorname{Var}_{i}\right) \quad \text { if } i \in I \backslash \bar{m},
\end{aligned}
$$

where $N_{i}, \operatorname{can}_{i}, \operatorname{Var}_{i}$ and $N$ are the abbreviation of $N_{i} \otimes \mathrm{id}$, etc. and $\operatorname{id} \otimes N$, and the action of $N$ on $C[\tau]$ is defined by $N \tau^{i}=i \tau^{i-1}, c f .2 .3$.

Proof. Put $D=g^{-1}(0)_{r e d}$, and let $j: U=X \backslash D \rightarrow X$ be the natural inclusion. Then by 2.3 and using the graph of $g, \psi_{\exp (2 \pi i \alpha)} K$ is represented by the mapping cone of

$$
j_{!}\left(j^{*} K \otimes_{c} E^{(\alpha)}\right) \rightarrow j_{*}\left(j^{*} K \otimes_{\boldsymbol{c}} E^{(\alpha)}\right)
$$

so that the action of $N$ on $\psi_{\exp (2 \pi i \alpha)} K$ corresponds to $\operatorname{id} \otimes N$. Here the pull back of $E^{(\alpha)}$ is also denoted by $E^{(\alpha)}$, and $-N \in$ End $E^{(\alpha)}$ is the logarithm of the unipotent part of the monodromy, divided by $2 \pi i$. By definition, the semisimple part of the monodromy of $E^{(\alpha)}$ around $D_{j}=\left\{x_{j}=0\right\}$ is the multiplication by $\exp \left(--2 \pi i \alpha m_{j}\right)$, and for $I \subset \bar{n} \backslash \bar{\nu}$ such that $I \cap \bar{m}=\emptyset$, we have a canonical isomorphism 


$$
\begin{aligned}
& \Psi_{\mathrm{I}}^{\nu} j_{!}\left(j^{*} K \otimes E^{(\alpha)}\right)=\Psi_{I}^{\nu} j_{*}\left(j^{*} K \otimes E^{(\alpha)}\right) \\
& \approx \Psi_{I}^{\nu+\alpha m} K \otimes i^{*} \mathbb{I}_{k \in \bar{m}} \psi_{x_{k,} \exp \left(-2 \pi j \alpha n_{k}\right)} E^{(\alpha)} \\
& \simeq \Psi_{I}^{\nu+\alpha m} K \otimes \mathbb{C}[\tau] \text { (cf. 2.3), }
\end{aligned}
$$

if we choose a factorization $\left(X^{\prime} \backslash D^{\prime}\right)^{\sim} \rightarrow \widetilde{S}^{*}$ of $\left(X^{\prime} \backslash D^{\prime}\right)^{\sim} \rightarrow X^{\prime} \backslash D^{\prime} \rightarrow S^{*}$ compatible with the base points. Here $X^{\prime}=\left\{x_{k}=0(k \notin \bar{m})\right\}, D^{\prime}=X^{\prime} \cap D, i: X^{\prime} \rightarrow X$, and we choose a base point of $\left(X^{\prime} \backslash D^{\prime}\right)^{\sim}$ such that $\operatorname{Re}(2 \pi i)^{-1} \log x_{k}=0$ on it. We check that the action of $N_{i}, \operatorname{can}_{i}(i \notin \bar{m}), \operatorname{Var}_{i}(i \notin \bar{m})$ on the first two terms correspond to $N_{i}-m_{i} N, \operatorname{can}_{i}, \operatorname{Var}_{i}$ on the last term. For $\nu \in(\mathbb{C} / \mathbb{Z})^{n}$ and $I \subset \bar{n} \backslash \bar{\nu}$, the morphisms

$$
\begin{aligned}
& \operatorname{can}_{I \cap \bar{m}}: \Psi_{I \backslash \bar{m}} j_{!}\left(j^{*} K \otimes E^{(\alpha)}\right) \rightarrow \Psi_{I}^{\nu} j_{!}\left(j^{*} K \otimes E^{(\alpha)}\right) \\
& \operatorname{Var}_{I \cap}-: \Psi_{I}^{\nu} j_{*}\left(j^{*} K \otimes E^{(\alpha)}\right) \rightarrow \Psi_{I \backslash}^{\nu} j_{*}\left(j^{*} K \otimes E^{(\alpha)}\right)
\end{aligned}
$$

are bijective, because

$$
\Psi_{I}^{\nu} j_{!}\left(j^{*} K \otimes E^{(\alpha)}\right)=\lim _{\rightarrow} \Psi_{I}^{\nu} j_{!}\left(j^{*} K \otimes E_{k}^{(\alpha)}\right)
$$

(same for $j_{*}$ ). Here $\operatorname{can}_{I}=\mathbb{H}_{i \in I} \operatorname{can}_{i}$, etc. Therefore the natural morphism

$$
\Psi_{I}^{\nu} j_{!}\left(j^{*} K \otimes E^{(\alpha)}\right) \rightarrow \Psi_{I}^{\nu} j_{*}\left(j^{*} K \otimes E^{(\alpha)}\right)
$$

is identified with

$$
N_{I \cap \bar{m}}: \Psi_{I \backslash \bar{m}}^{\nu} j_{*}\left(j^{*} K \otimes E^{(\alpha)}\right) \rightarrow \Psi_{I \backslash \bar{m}}^{\nu} j_{*}\left(j^{*} K \otimes E^{(\alpha)}\right),
$$

and then with

$$
\left(N_{*}-m_{*} N\right)_{I \cap \bar{m}}: \Psi_{I \backslash \bar{m}}^{\nu+\alpha m} K \otimes \mathbb{C}[\tau] \rightarrow \Psi_{I \backslash \bar{m}}^{\nu+\alpha} K \otimes \mathbb{C}[\tau] .
$$

We check that this morphism is surjective, using a filtration on $\Psi_{I \backslash \bar{m}}^{\nu+\alpha m} K$ and reducing to the case where $N_{i}=0$ on $\Psi_{I \backslash \bar{m}}^{y+\alpha} K$ for $i \in \bar{m}$. Thus we get the first assertion and the other assertions follow from the above description of the action of $N_{i}$, etc. on $\Psi_{I}^{\nu} j_{!}\left(j^{*} K \otimes E^{(\alpha)}\right)$, etc.

3.3. Corollary。 For $\nu, I$ and $K$ as in 3.2, we have a functorial isomorphism as $\mathbb{C}[N]$-modules:

$\Psi_{I}^{\nu}{ }^{p} \psi_{g, \exp (2 \pi i \alpha)} K=\operatorname{Coker}\left(\left(N_{*}-m_{*} N\right)_{I \cap \bar{m}}: \Psi_{I \backslash \bar{m}}^{\nu+\alpha^{m}} K \otimes \mathbb{C}[N] \rightarrow \Psi_{I \backslash \bar{m}}^{\nu+\alpha_{m}} K \otimes \mathbb{C}[N]\right)$ such that $N_{i}, \operatorname{can}_{i}, \operatorname{Var}_{i}$ on the left hand side correspond respectively to the morphisms defined by the morphisms in 3.2 .

Proof. With the notations of 2.3, we have an exact sequence of left $W$-modules:

$$
0 \rightarrow W / W \tau \stackrel{\circ}{\rightarrow} W / W \tau s \rightarrow W / W s \rightarrow 0
$$


Substituting $W / W \tau=\boldsymbol{C}[s], W / W \tau s=\boldsymbol{C}\left[s, s^{-1}\right], W / W s=\boldsymbol{C}[\tau]$ as $\boldsymbol{C}[N]$-modules, we get

$$
0 \rightarrow \boldsymbol{C}[s] \rightarrow \boldsymbol{C}\left[s, s^{-1}\right] \rightarrow \boldsymbol{C}[\tau] \rightarrow 0
$$

Put $H=\Psi_{I \backslash \bar{m}}^{\nu+m^{m}} K$ as a $\boldsymbol{C}\left[N_{1}, \cdots, N_{n}\right]$-module, and $H[s]=H \otimes \boldsymbol{C}[s]$ (same for $\left.\left[s, s^{-1}\right],[\tau]\right)$. Then we have a commutative diagram

$$
\begin{gathered}
0 \rightarrow H[s] \rightarrow H\left[s, s^{-1}\right] \rightarrow H[\tau] \rightarrow 0 \\
\downarrow \tilde{N}_{J} \quad \downarrow \tilde{N}_{J} \quad \downarrow \tilde{N}_{J} \\
0 \rightarrow H[s] \rightarrow H\left[s, s^{-1}\right] \rightarrow H[\tau] \rightarrow 0,
\end{gathered}
$$

where $\tilde{N}_{J}=\left(N_{*}-m_{*} N\right)_{J}$ with $J=I \cap \bar{m}$, and $N=s$. We check that the middle vertical morphism $\tilde{N}_{J}$ is bijective, and get

$$
\operatorname{Ker}\left(\tilde{N}_{J}: H[\tau] \rightarrow H[\tau]\right)=\operatorname{Coker}\left(\tilde{N}_{J}: H[s] \rightarrow H[s]\right),
$$

which proves the assertion.

3.4. Remark. The above isomorphism in 3.3 is compatible with the analytic one in [S2, 3.4]. In fact, with the notations of [loc. cit], the isomorphism in 3.2 corresponds to the isomorphism in the derived category of $\mathscr{D}_{X}$-Modules with the action of $N$ :

$$
\begin{aligned}
\psi_{\exp (2 \pi i \alpha)} M & =\left[j_{!}\left(j^{*} M \otimes t^{\alpha} \boldsymbol{C}[\tau]\right) \rightarrow j_{*}\left(j^{*} M \otimes t^{\alpha} \boldsymbol{C}[\tau]\right)\right] \\
& =\operatorname{Ker}\left(j_{!}\left(j^{*} M \otimes t^{\alpha} \boldsymbol{C}[\tau]\right) \rightarrow j_{*}\left(j^{*} M \otimes t^{\alpha} \boldsymbol{C}[\tau]\right)\right)
\end{aligned}
$$

for $-1 \leqq \alpha<0$ (cf 2.3), using the graph of $g$ and then the projection $X \times \boldsymbol{C} \rightarrow X$ (i.e. taking $D R_{S}$ ), where $j_{!}, j_{*}$ are taken in the category of inductive limit of regular holonomic quasi-unipotent $\mathscr{D}$-Modules with normal crossing singular supports, i.e.

$$
j_{*}\left(j^{*} M \otimes t^{\alpha} C[\tau]\right)=\lim _{\rightarrow} j_{*}\left(j^{*} M \otimes t^{\alpha}\left(\oplus_{i \leqq k} C \tau^{i}\right)\right) .
$$

Here $t^{\alpha}, \tau=\log t$ mean $\tilde{e}_{\alpha}, \tilde{\tau}$ in 2.3 so that

$$
x_{i} \partial_{i} t^{\alpha}=m_{i} \alpha t^{\alpha}, \quad \partial_{i} \tau^{j}=m_{i} j \tau^{j-1}
$$

with $\partial_{i}=\partial / \partial x_{i}$, and $(u \otimes P) \partial_{i}=u \partial_{i} \otimes P-u \otimes \partial_{i} P$ for $u \in M, P \in t^{\alpha} \mathbb{C}[\tau]$. In fact, the first isomorphism in (3.4.1) is induced by the morphisms of functors

$$
\mathrm{id} \leftarrow D R_{S}\left(i_{g}\right)_{*} \leftarrow V_{0} D R_{S}\left(i_{g}\right)_{*} \rightarrow G r_{0}^{V} D R_{S}\left(i_{g}\right)_{*}
$$

applied to $\left[\tilde{M}_{!}^{\alpha} \rightarrow \tilde{M}_{*}^{\alpha}\right]$, where $\tilde{M}_{!}^{\alpha}=j_{!}\left(j^{*} M \otimes t^{\alpha} \mathbb{C}[\tau]\right)$ (same for $\left.\tilde{M}_{*}^{\alpha}\right)$ and $\psi_{\exp (2 \pi i \alpha)}$ $M=G r_{a}^{V} M\left[\partial_{t}\right]$ with $M\left[\partial_{t}\right]=\left(i_{g}\right)_{*} M$, cf. $[\mathrm{S} 1,3.4 .12]$. Here 


$$
V_{\alpha} D R_{S}=C\left(\partial_{t}: V_{\alpha-1} \rightarrow V_{\alpha}\right),
$$

and $G r_{0}^{V} D R_{S}\left(i_{g}\right)_{*} M_{!}^{\alpha}=0$, because $\left(i_{g}\right)_{*}$ commutes with $j_{!}$if the inclusion $X \times$ $\mathbb{C}^{*} \rightarrow X \times \mathbb{C}$ is also denoted by $j$. We use the natural projection (after multiplied by $t^{-\infty-1}$ [loc. cit]):

$$
\left[G r_{-1}^{V} \tilde{M}_{*}^{\alpha}\left[\partial_{t}\right] \stackrel{\partial_{t}}{\rightarrow} G r_{0}^{V} \tilde{M}_{*}^{\alpha}\left[\partial_{t}\right]\right] \rightarrow G r_{a}^{V} M\left[\partial_{t}\right]
$$

such that the action of $N$ on the left hand side corresponds to that of $s-\alpha$ on the right, where $\tilde{M}_{*}^{\alpha}\left[\partial_{t}\right]=\left(i_{g}\right)_{*} \tilde{M}_{*}^{\alpha}$ (same for $\tilde{M}_{!}^{\alpha}\left[\partial_{t}\right]$ ). Let Ker be the last term of (3.4.1). We define a $\mathscr{D}_{X}[N]$-linear morphism by

$$
\operatorname{Ker} \ni u \mapsto \sum(-1)^{i} u g^{i} / i ! \otimes \partial_{t}^{i} \in \tilde{M}_{!}^{\alpha}\left[\partial_{t}\right]
$$

Then this gives a commutative diagram

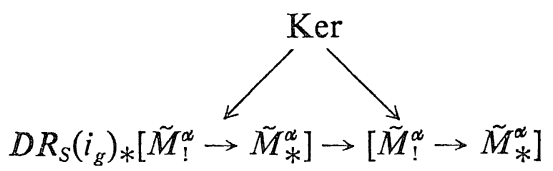

of $\mathscr{D}_{X}[N]$-Modules.

Now let $\left.u^{\prime}=u \partial_{J} \in(\operatorname{Ker})^{\nu+1} I \subset\left(\tilde{M}_{!}^{\alpha}\right)^{\nu+1} I\right)$ for $\nu \in[-1,0)^{n}$ and $I \subset\left\{i \in \bar{n}: \nu_{i}=\right.$ $-1\}$, where $J=I \cap \bar{m}$ and $u \in\left(\tilde{M}_{!}^{\alpha}\right)^{\nu+1} I \backslash \bar{m}$. Then $u^{\prime}$ is annihilated by $g$, and the image of $u^{\prime}$ by (3.4.3) is $u^{\prime} \otimes 1=u \partial_{J} \otimes 1 \in \tilde{M}_{!}^{\alpha}\left[\partial_{t}\right]$. We define

$$
v=\left(u \partial_{J} \otimes 1-(u \otimes 1) \partial_{J}\right) \partial_{t}^{-1} \in G r_{-1}^{V} \tilde{M}_{!}^{\alpha}\left[\partial_{t}\right],
$$

using $\left(u \otimes \partial_{t}^{i}\right) \partial_{j}=u \partial_{j} \otimes \partial_{t}^{i}-u\left(\partial_{j} g\right) \otimes \partial_{t}^{j+1}$. Then $v \partial_{t}=u^{\prime} \otimes 1$ in $G r_{0}^{V} \tilde{M}_{!}^{\alpha}\left[\partial_{t}\right]$, because $u \otimes 1 \in V_{<0} \tilde{M}_{1}^{\alpha}\left[\partial_{t}\right]$. Let $v_{1}$ be the image of $v$ in $G r_{-1}^{V} M_{*}^{\alpha}\left[\partial_{t}\right]$, and $v_{2}$ that of $v_{1}$ by the projection (3.4.2). Put

$$
H=M^{\nu+1_{\nearrow} \backslash \bar{m}+\alpha m} .
$$

Then we have the isomorphism

$$
(\mathrm{Ker})^{y+1}{ }_{I}=\operatorname{Ker}\left(\tilde{N}_{J}: H[\tau] \rightarrow H[\tau]\right)
$$

where $\tilde{N}_{J}$ is as in the proof of 3.3 , and $u$ belongs to the right hand side of the above isomorphism. By definition, $u$ corresponds to $v_{2}$ in $G r_{a}^{V} M\left[\partial_{t}\right]$, and we check that by the isomorphism (cf. [S2, 3.4]):

$$
\left(G r_{a}^{V} M\left[\partial_{t}\right]\right)^{v+1}=\operatorname{Coker}\left(\tilde{N}_{J}: H[N] \rightarrow H[N]\right),
$$

$v_{2}$ coincides up to sign with the image of $u$ by the isomorphism used in the proof 
of 3.3. In fact $v_{2}$ is the projection of $-\left(\tilde{N}_{J} u\right) s^{-1} \in H[\tau, s]$ into $H[s]=H[\tau, s] /$ $H[\tau, s] \tau$, where $[s, \tau]=1$ and $N_{i}$ is the left multiplication of $N_{i}-m_{i} s$. This proves the assertion.

The following fact was found by Kashiwara in the unipotent and reduced case.

3.5. Proposition. Let $X$ and $g$ be as above, and $\mathbf{S}: K \otimes L \rightarrow T_{X}$ a pairing of $K$, $L \in \operatorname{Perv}\left(\boldsymbol{C}_{X}\right)_{n c}$. Then by the isomorphism in 3.3, the induced pairing

$$
\Psi_{I}^{\nu}{ }^{p} \psi S: \Psi_{I}^{\nu} \psi_{\exp (2 \pi i \alpha)} K \otimes \Psi_{I}^{-\nu}{ }^{p} \psi_{\exp (-2 \pi i \alpha)} L \rightarrow C
$$

is identified with $\operatorname{Res} \Psi_{I \backslash \bar{m}}^{\nu+\alpha m} \mathbf{S} \circ\left(N_{I \cap \bar{m}}^{-1} \otimes \mathrm{id}\right)$, where $\Psi_{I \backslash \bar{m}}^{\nu+\alpha m} \mathbf{S}$ is extended to

$$
\Psi_{I \backslash \bar{m}}^{\nu+\alpha m} \mathbf{S}: \Psi_{I \backslash \bar{m}}^{\nu+\alpha m} K\left[N, N^{-1}\right] \otimes \Psi_{I \backslash \bar{m}}^{-\nu-\alpha m} L\left[N, N^{-1}\right] \rightarrow \boldsymbol{C}\left[N, N^{-1}\right]
$$

by $\quad \Psi_{I \backslash \bar{m}}^{\nu+\alpha m} \mathbf{S}\left(u \otimes N^{i}, v \otimes N^{j}\right)=(-1)^{i} \Psi_{I \backslash \bar{m}}^{\nu+\alpha m} \mathbf{S}(u, v) \otimes N^{i+j}$ for $u \in \Psi_{I \backslash \bar{m}}^{\nu+\alpha m} K, v \in$ $\Psi_{I \backslash \bar{m}}^{-\nu-\alpha m} L$, and Res: $\boldsymbol{C}\left[N, N^{-1}\right] \rightarrow C$ is defined by $\operatorname{Res}\left(\sum a_{i} \otimes N^{i}\right)=a_{-1}$.

Proof. We assume that $K, L$ and $T_{X}$ are represented by complexes of flasque sheaves, and $\mathbf{S}$ by a morphism of complexes. As in 2.3 , the local systems on $S^{*}$ such that the semisimple part of its monodromy is the multiplication by $\exp (-2 \pi i \alpha)$ is identified with the $C[N]$-modules, by using the functor: $E \mapsto$ $\Gamma\left(\tilde{S}^{*}, \pi^{*} E\right)$. Put $\hat{W}=\boldsymbol{C} \llbracket s \rrbracket[\tau]$ with $[s, \tau]=1$. We consider the limit of local

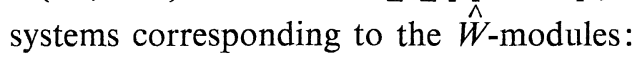

$$
e_{\alpha} \boldsymbol{C} \llbracket s \rrbracket, e_{\alpha} \boldsymbol{C}[\tau], e_{\alpha} \boldsymbol{C} \llbracket s \rrbracket\left[s^{-1}\right],
$$

and they are denoted also by the same symbols, where

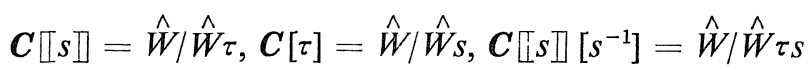

with $s=N$, and $e_{\alpha}$ means the condition on the semisimple part of the monodromy, cf. 2.3. Let $D, U, j$ be as in the proof of 3.2. We define

$$
K_{*}^{\alpha} \llbracket s \rrbracket\left[s^{-1}\right]=j_{*}\left(j^{*} K \otimes e_{\alpha} \boldsymbol{C} \llbracket s \rrbracket\left[s^{-1}\right]\right) \text {, etc . }
$$

to be the inductive limit (with respect to $\left[s^{-1}\right],[\tau]$ ) of the projective limit (with respect to $\llbracket s \rrbracket$ ). (It is same for $K_{!}^{\alpha} \llbracket s \rrbracket\left[s^{-1}\right]$, etc.) Then using a condition of Mittag-Leffler, we have an exact sequence of complexes:

$$
0 \rightarrow K_{*}^{\alpha} \llbracket\left[s \rrbracket \rightarrow K_{*}^{\alpha} \llbracket s \rrbracket\left[s^{-1}\right] \rightarrow K_{*}^{\alpha}[\tau] \rightarrow 0 \quad \text { (same for } K_{!}^{\alpha}\right. \text { ) . }
$$

We have also a multiplication of limits of local systems:

$$
e_{\alpha} \boldsymbol{C}[\tau] \otimes e_{\beta} \boldsymbol{C} \llbracket s \rrbracket\left[s^{-1}\right] \rightarrow e_{\alpha+\beta} \boldsymbol{C} \llbracket s_{\rrbracket}\left[s^{-1}\right]
$$


(same for $\left.e_{\beta} \mathbb{C} \llbracket s \rrbracket, e_{\beta} \mathbb{C}[\tau]\right)$, because $\mathbb{C} \llbracket s \rrbracket\left[s^{-1}\right]$ is a $\hat{W}$-modules and $N\left(\tau^{i} u\right)=i \tau^{i-1}$ $u+\tau^{i} N u$. Therefore we get

$$
K_{*}^{\alpha}[\tau] \otimes L_{*}^{-\alpha} \llbracket s \rrbracket\left[s^{-1}\right] \rightarrow\left(T_{X}\right)_{*}^{0} \llbracket s \rrbracket\left[s^{-1}\right], \text { etc. }
$$

In particular, we get the induced pairing:

$$
\psi \mathbb{S}: i^{*} K_{*}^{\alpha}[\tau] \otimes i^{*} L_{*}^{-\alpha}[\tau] \rightarrow\left[i^{*} T_{X} \rightarrow i *\left(T_{X}\right)_{*}^{0}[\tau] \stackrel{-N}{\rightarrow} i^{*}\left(T_{X}\right)_{*}^{0}[\tau]\right]
$$

by 2.3 , where $i^{*} T_{X}$ in the last term is at degree -2 . We check that this morphism is naturally extended to:

$$
\begin{aligned}
\psi & S: C\left(K_{!}^{\alpha}[\tau] \rightarrow K_{*}^{\alpha}[\tau]\right) \otimes C\left(L_{!}^{-\alpha}[\tau] \rightarrow L_{*}^{-\alpha}[\tau]\right) \\
& \rightarrow C\left(\left[\left(T_{X}\right)_{!} \rightarrow\left(T_{X}\right)_{!}^{0}[\tau] \stackrel{-N}{\rightarrow}\left(T_{X}\right)_{!}^{0}[\tau]\right] \rightarrow\left[T_{X} \rightarrow\left(T_{X}\right)_{*}^{0}[\tau] \stackrel{-N}{\rightarrow}\left(\mathbb{I}_{X}\right)_{*}^{0}[\tau]\right]\right)
\end{aligned}
$$

by 3.6 below, where $\left(T_{X}\right)_{!}=j_{!} j^{*} T_{X}$. Similarly we have

$$
\begin{aligned}
& C\left(K_{!}^{\alpha}[\tau] \rightarrow K_{*}^{\alpha}[\tau]\right) \otimes C\left(L_{!}^{-\alpha}((s)) \rightarrow L_{*}^{-\alpha}((s))\right) \\
& \quad \rightarrow C\left(C\left(\left(T_{X}\right)_{1}^{0}((s)) \stackrel{-N}{\rightarrow}\left(T_{X}\right)_{!}^{0}((s))\right) \rightarrow C\left(\left(T_{X}\right)_{*}^{0}((s)) \stackrel{-N}{\rightarrow}\left(T_{X}\right)_{*}^{0}((s))\right)\right)
\end{aligned}
$$

compatible with the natural morphisms $L_{!}^{-\infty}((s)) \rightarrow L_{!}^{-\infty}[\tau]$, etc., where $L_{!}^{-\infty}((s))=$ $L_{!}^{-\alpha} \llbracket s \rrbracket\left[s^{-1}\right]$, etc. Then by 3.6 (where $A=0$ ), we get a pairing $\psi \mathbb{S}$ of $C\left(K_{1}^{\alpha}[\tau]\right.$ $\left.\rightarrow K_{*}^{\alpha}[\tau]\right)$ and the mapping cone of the surjective morphism:

$$
C\left(L_{!}^{-\alpha}((s)) \rightarrow L_{*}^{-\alpha}((s))\right) \rightarrow C\left(L_{!}^{-\alpha}[\tau] \rightarrow L_{*}^{-\alpha}[\tau]\right)
$$

with value in the mapping cone of the surjective morphism of the mapping cone of

$$
\begin{aligned}
& C\left(\left(T_{X}\right)_{!} \oplus\left(T_{X}\right)_{!}^{0}((s)) \stackrel{(0,-N)}{\longrightarrow}\left(T_{X}\right)_{!}^{0}((s))\right) \\
& \quad \rightarrow C\left(T_{X} \oplus\left(T_{X}\right)_{*}^{0}((s)) \stackrel{(0,-N)}{\longrightarrow}\left(T_{X}\right)_{*}^{0}((s))\right)
\end{aligned}
$$

into the mapping cone of

$$
C\left(\left(T_{X}\right)_{!}^{0}[\tau] \stackrel{-N}{\rightarrow}\left(T_{X}\right)_{!}^{0}[\tau]\right) \rightarrow C\left(\left(T_{X}\right)_{*}^{0}[\tau] \stackrel{-N}{\rightarrow}\left(T_{X}\right)_{* *}^{0}[\tau]\right) .
$$

Therefore we get the pairing $\psi \mathbb{S}$ of

$$
C\left(K_{!}^{\alpha}[\tau] \rightarrow K_{*}^{\alpha}[\tau]\right) \text { and } C\left(L_{!}^{-\alpha} \llbracket s \rrbracket \rightarrow L_{*}^{-\alpha} \llbracket s \rrbracket\right)[1]
$$

with value in the kernel of the above surjective morphism of the mapping cones. We check that the kernel is the mapping cone of 


$$
\begin{aligned}
& C\left(\left(T_{X}\right)_{1} \oplus\left(T_{X}\right)_{1}^{0} \llbracket s \rrbracket \stackrel{(-\iota,-N)}{\longrightarrow}\left(T_{X}\right)_{1}^{0} \llbracket s \rrbracket\right)[1] \\
& \quad \rightarrow C\left(T_{X} \oplus\left(T_{X}\right)_{*}^{0} \llbracket s \rrbracket \stackrel{(\iota,-N)}{\longrightarrow}\left(T_{X}\right)_{*}^{0}[s \rrbracket)[1]\right.
\end{aligned}
$$

where the morphism is defined by the mapping cone of $(p,-\varepsilon ;-\varepsilon)$, and $\iota, p$ and $\varepsilon$ denote the natural morphisms. By definition $\psi \mathbb{S}$ factors through the mapping cone of

$$
C\left(0 \rightarrow ( T _ { X } ) _ { 1 } ^ { 0 } \left[[s \rrbracket)[1] \rightarrow C\left(0 \rightarrow\left(T_{X}\right)_{*}^{0}[s]\right)[1]\right.\right.
$$

and it is homotopic to the morphism (also denoted by $\psi \mathbb{S}$ ) which factors through

$$
C\left(T_{X} \oplus\left(T_{X}\right)_{*}^{0} \llbracket s \rrbracket \stackrel{(\ell,-N)}{\longrightarrow}\left(T_{X}\right)_{*}^{0} \llbracket[s \rrbracket)[1],\right.
$$

because $(-\imath,-N)$ in the first mapping cone is an isomorphism of complexes. Combining with the natural projection to $T_{X}$ [2], we get the pairing

$$
\psi \mathbb{S}: C\left(K_{!}^{\alpha}[\tau] \rightarrow K_{*}^{\infty}[\tau]\right) \otimes C\left(L_{1}^{-\alpha} \llbracket\left[s \rrbracket \rightarrow L_{*}^{-\alpha} \llbracket s \rrbracket\right)[1] \rightarrow T_{X}[2],\right.
$$

whose restriction to $K_{!}^{\alpha}[\tau][1] \otimes L_{*}^{-\alpha}[s][1]$ is the composition:

$$
\begin{aligned}
& \left(K_{!}^{\alpha}[\tau]\right)[1] \otimes\left(L_{*}^{-\alpha} \llbracket s \rrbracket\right)[1]=\left(K_{!}^{\alpha}[\tau] \otimes L_{*}^{-\alpha} \llbracket s \rrbracket\right)[2] \\
& \stackrel{S}{\rightarrow}\left(T_{X}\right)_{!} \llbracket s \rrbracket[2] \rightarrow\left(T_{X}\right)_{!}[2] \rightarrow T_{X}[2]
\end{aligned}
$$

by 3.6 , where the first isomorphism is induced by

$$
K_{!}^{\alpha}[\tau] \otimes \mathbb{C}[1]=\mathbb{C}[1] \otimes K_{!}^{\alpha}[\tau]
$$

and the second morphism is defined as above (using $\hat{W}$-module structure). Here note that the projection of (3.5.1) onto $T_{X}$ [2] represents the natural morphism: $i_{*} i^{l} T_{X}[2] \rightarrow \mathbb{T}_{X}[2]$. By definition, the restriction of

$$
{ }^{p} \psi \mathbb{S}: \mathcal{C}\left(K_{!}^{\alpha}[\tau] \rightarrow K_{*}^{\alpha}[\tau]\right)[-1] \otimes C\left(L_{!}^{-\alpha} \llbracket s \rrbracket \rightarrow L_{*}^{-\alpha} \llbracket s \rrbracket\right) \rightarrow T_{X}
$$

to $K_{!}^{\alpha}[\tau] \otimes L_{*}^{-\alpha}[[s]$ is the composition:

$$
K_{!}^{\alpha}[\tau] \otimes L_{*}^{-\alpha}\left[s \rrbracket \stackrel{-S}{\rightarrow}\left(T_{X}\right)_{!} \llbracket s \rrbracket \rightarrow\left(T_{X}\right)_{!} \rightarrow T_{X} .\right.
$$

Put $H=\Psi_{I \backslash \bar{m}}^{\nu+a_{m}} K, H^{\prime}=\Psi_{I \backslash m}^{-\nu-\alpha m} \bar{L}$ so that

$$
\begin{aligned}
& \Psi_{I}^{\nu}{ }^{p} \psi_{g, \exp (2 \pi i \omega)} K=\mathbb{K} \operatorname{er}\left(\tilde{N}_{J}: H[\tau] \rightarrow H[\tau]\right) \\
& \Psi_{I}^{-\nu p} \psi_{g, \exp (-2 \pi i \omega)} L=\operatorname{Coker}\left(\tilde{N}_{J}: H^{\prime} \llbracket s \rrbracket \rightarrow H^{\prime} \llbracket s \rrbracket\right)
\end{aligned}
$$

by $3.2-3$ (using the bijectivity of $\tilde{N}_{J}: H^{\prime}((s)) \rightarrow H^{\prime}((s))$ ). Then the pairing 
$\Psi_{I}^{y}{ }^{p} \psi \mathbf{S}$ of these two vector spaces are induced by

$$
-\Psi_{I \backslash \bar{m}}^{\nu+\alpha_{m}} \mathbf{S}: H[\tau] \otimes H^{\prime} \llbracket s \rrbracket \rightarrow \mathbb{C} \llbracket s \rrbracket \rightarrow \mathbb{C}
$$

where $-\Psi_{I \backslash \bar{m}}^{\nu+\alpha m} \mathbf{S}\left(u \otimes \tau^{i}, v \otimes s^{j}\right)=(-1)^{i-1} i ! \Psi_{I \backslash \bar{m}}^{\nu+a_{m}} \mathbf{S}(u, v)$ (if $\left.i=j\right), 0$ (otherwise) for $u \in H, v \in H^{\prime}$. Therefore it is enough to show that this pairing coincides with the pairing in the assertion by the isomorphism in the proof of 3.3. We first check that the image of $u \otimes s^{-j-1} \in H((s))$ in $H[\tau]$ is $u \otimes \tau^{j} / j$ !, because $\tau^{j}\left(s^{-1}\right)=j ! s^{-j-1}$ in $\boldsymbol{C}((s))$. Then the assertion follows from the commutative diagram:

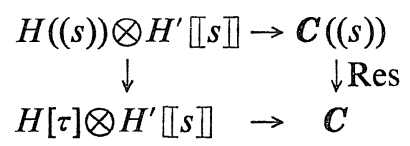

where the first horizontal morphism is defined by $\Psi \boldsymbol{S}$ as in the assertion, and the second by $-\Psi \boldsymbol{S}$ as above. In fact, $\Psi_{I}^{\nu}$ of the isomorphism

$$
C\left(L_{!}^{-\infty}[\tau] \rightarrow L_{*}^{-\infty}[\tau]\right) \stackrel{\sim}{\rightarrow} C\left(L_{!}^{-\infty} \llbracket s \rrbracket \rightarrow L_{*}^{-\alpha}[s \rrbracket)[1] \text { in } D_{c}^{b}\left(C_{X}\right)\right.
$$

coincides with the morphism used in the proof of 3.3 shifted by 1 .

3.6 Lemma. Let $f: A \otimes D \rightarrow E, g: B \otimes C \rightarrow E, h: B \otimes D \rightarrow F$ be morphisms of complexes compatible with morphisms $u: A \rightarrow B, v: C \rightarrow D, w: E \rightarrow F$, i.e. $f \circ(\mathrm{id} \otimes v)$ $=g \circ(u \otimes \mathrm{id}), h \circ(\mathrm{id} \otimes v)=w g, h \circ(u \otimes \mathrm{id})=w f$. Then we have a morphism of complexes

$$
C(u: A \rightarrow B) \otimes C(v: C \rightarrow D) \rightarrow C(w: E \rightarrow F)
$$

defined by $(a, b ; c, d) \mapsto\left(f(a, d)+(-1)^{i} g(b, c), h(b, d)\right)$ for $a \in A^{i+1}, b \in B^{i}$, $c \in C^{j+1}, d \in D^{j}$.

(The proof is left to the reader.)

3.7. Remark. Using 3.3-5, we can generalize [S2, 3.20, 3.27] to the nonquasiunipotent case where the filtration $V$ is indexed by $\boldsymbol{R}$. Note that the absolute value of the eigenvalues of the local monodromies of the polarizable variations of Hodge structures is 1 , and the other results in [S1, 2] can be generalized to this case, because the result of Schmid and Zucker used in [S1] is true in this case by Deligne-Schmid and Zucker.

\section{References}

[BBD] Beilinson, A.A., Bernstein, J., Deligne, P., Faisceaux pervers, Astérisque, 100 (1982), 
5-171.

[D1] Deligne, P., Le formalisme des cycles évanescents, in SGA7, Lect. Note Math., 340 Springer (1973), 82-115.

[D2] - Cohomologie à supports propres, in SGA 4, Lect. Note Math., 305 (1973), 250-461.

[K1] Kashiwara, M., B-function and holonomic systems, Invent. Math., 38 (1976), 3353.

[K2] - Vanishing cycle sheaves and holonomic systems of differeitial equations, in Lect. Note Math., 1016 Springer (1983), 134-142.

[K3] - On the holonomic systems of linear differential equations II, Inv. Math., 49 (1978), 121-135.

[K4] - On the maximally overdetermined systems of linear differential equations I, Publ. RIMS, 10 (1975), 563-579.

[M] Malgrange, B., Polynôme de Bernstein-Sato et cohomologie évanescente, Astérisque, 101-102 (1983), 243-267.

[S1] Saito, M., Modules de Hodge polarisables, Publ. RIMS, 24 (1988), 849-995.

[S2] — - Mixed Hodge Modules, preprint RIMS-585, July, 1987.

[S3] — On the structure of Brieskorn lattices, preprint Grenoble Sept. 1983. 
